\title{
ESTUDO SOBRE PARCELAS PERDIDAS NO DELINEAMENTO EM QUADRADO DE YOUDEN
}

\author{
MARINÉIA DE LARA HADDAD \\ Engenheira-Agrônoma
}

Orientador: Dr. IZAIAS RANGEL NOGUEIRA

Tese apresentada à Escola Superior de Agricultura "Luiz de Queiroz", para obtenção do título de Doutor em Agronomia. Área de Concentração: Estatística e Experimentação Agronômica.

$P \mid R A C I C A B A$

Estado de São Paulo - Brasil

Maio - 1982 
ii.

Aos meus pais, Paulo e Neja, Ao meu esposo Cláudio, Aos meus filhos, Cecíita e Cláudio, $D E D I C O$ 
iii.

\section{AGRADECIMENTOS}

Ao Dr. Izaias Rangel Nogueira, Professor Titular (aposentado) do Departamento de Matemática e Estatística da ESALQ, pela orientação segura, o qual, mais do que orientador, foi amigo.

Á Empresa Brasileira de Pesquisa Agropecuária (EMBRAPA), pela oportunidade oferecida para a realização do Curso de Pós-Graduação.

Ao Prof. Dr. Humberto de Campos, Coordenador do Curso de Pós-Graduação em Estatística e Experimentação Agronômica, pelas sugestões apresentadas.

Aos demais professores e funcionārios do Departamento de Matemática e Estatística da ESALQ, pelos ensinamentos e constante disponibilidade.

Ao Prof. Dr. Irineu Umberto Packer, Docente-Livre do Departamento de Zootecnia da ESALQ, pela versão do Resumo para o Inglềs.

Aos colegas do Curso de Pós-Graduação, pelo espírito de solidariedade e companheirismo.

A Srta. Maria Izalina Ferreira Alves, Secretāria do Departamento de Matemática e Estatística da ESALQ, pelo trabalho datilogrāfico.

A todos que, de uma forma ou de outra, contribuiram para a realização deste trabalho. 


\section{I $N D$ I C E}

Pág.

RESUMO

$\mathrm{x}$

SUMMARY

xii

1. INTRODUÇÃO $\quad \ldots \ldots \ldots \ldots \ldots \ldots \ldots \ldots \ldots \ldots \ldots \ldots \ldots \ldots \ldots \ldots$

2. REVISÃO DE LITERATURA $\ldots \ldots \ldots \ldots \ldots \ldots \ldots \ldots \ldots \ldots \ldots \ldots \ldots \ldots \ldots \ldots$

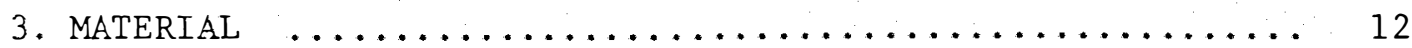

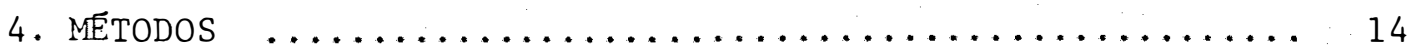

4.1 - Modelo Matemático ...................... 15

4.2 - Sistema de Equações Normais .............. 16

4.3 - Sistema de Equações Normais Para o Caso de Perda de Uma ou de Duas Parcelas na Mesma Linha ......... 17

4.4 - Estimativas dos Efeitos dos Parâmetros ......... 18

4.5 - Cálculo das $\operatorname{SQP}\left(\hat{l}_{k}, \hat{c}_{j}, \hat{t}_{i(j, k)}, \operatorname{SQP}\left(\tilde{l}_{k}, \tilde{c}_{j}\right)\right.$ e $\operatorname{SQP}\left(\underline{\underline{c}}_{j}\right) 19$

4.6 - Estimativas dos Efeitos de Tratamentos $\hat{\tau}$, Através do Sistema Reduzido......................... 22

4.7 - Estimativa de Uma Parcela Perdida ............. 24

4.8 - Estimativas de Duas Parcelas Perdidas na Mesma Linha 25

4.9 - Correção das Somas de Quadrados, Levando-se em Consi deração a Estimativa de Uma Parcela Perdida .... 26

4.10 - Correção das Somas de Quadrados, Levando-se em Consi deração as Estimativas de Duas Parcelas Perdidas .. 29

4.11 - Verdadeiras Somas de Quadrados .............. 30

4.12 - Matriz de Dispersão ...................... 35

4.13 - Variância da Estimativa de Um Contraste Entre Duas Mé dias de Tratamentos Ajustadas para Linhas e Colunas 36 
5. RESULtados E DiscUSSÃo $\ldots \ldots \ldots \ldots \ldots \ldots \ldots \ldots \ldots \ldots \ldots \ldots$

5.1 - Caso de Uma Parcela Perdida, Ignorando-se a Mesma . 40

5.1.1 - Sistema de equações normais e estimativas dos parâmetros $\ldots \ldots \ldots \ldots \ldots \ldots \ldots \ldots \ldots \ldots \ldots$. 40

5.1 .2 - Cálculo das $\mathrm{SQP}_{(1)}, \mathrm{SQP}_{(2)}$ e $\mathrm{SQP}_{(3)} \ldots \ldots .42$

5.1 .3 - Análise da variância $\ldots \ldots \ldots \ldots \ldots \ldots \ldots \ldots 43$

5.1.4 - Variância da estimativa de um contraste entre duas médias de tratamentos, ajustadas pa ra linhas e colunas ................ 44

5.2 - Caso de Uma Parcela Perdida, Considerando-se a Sua Estimativa $\ldots \ldots \ldots \ldots \ldots \ldots \ldots \ldots \ldots \ldots \ldots$

5.2.1 - Fórmula para a estimativa de uma parcela per dida $\ldots \ldots \ldots \ldots \ldots \ldots \ldots \ldots \ldots \ldots$

5.2.2 - Fórmulas para as correções das somas de quadrados de tratamentos e de linhas: $U_{T}$ e $U_{L} \cdots \quad 46$

5.2 .3 - Anālise da variância ............. 49

5.2.4 - Comparação entre duas médias de tratamentos, ajustadas para linhas e colunas .........

5.3 - Caso de Duas Parcelas Perdidas na Mesma Linha, Sendo que o Tratamento Correspondente à Parcela Perdida de Uma Coluna Não Figure Dentre as Parcelas Remanescentes da Outra Coluna em que Ocorreu a Perda e Vice-Ver sa. As Parcelas Perdidas São Ignoradas ..........

5.3.1 - Sistema de equações normais e estimativas dos

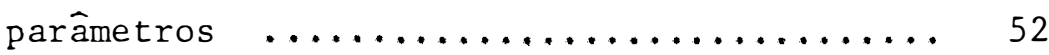

5.3 .2 - Cálculo das $\mathrm{SQP}_{(1)}, \mathrm{SQP}_{(2)}$ e $\mathrm{SQP}_{(3)} \ldots \ldots \ldots 54$ 
vi.

5.3 .3 - Anālise da variância ................ 54

5.3.4 - Variância da estimativa de um contraste entre duas médias de tratamentos, ajustadas pa ra linhas e colunas $\ldots \ldots \ldots \ldots \ldots \ldots \ldots$

5.4 - Caso de Duas Parcelas Perdidas na Mesma Linha, sendo que o Tratamento Correspondente à Parcela Perdida de Uma Coluna Não Figure Dentre as Parcelas Remanescentes da Outra Coluna em que Ocorreu a Perda e Vice-Versa. As Estimativas das Parcelas São Consideradas

5.4.1 - Fórmulas para as estimativas das parcelas per

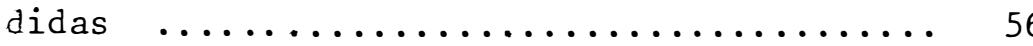

5.4.2 - Fórmulas para as correções das somas de quadrados de tratamentos e de linhas: $U_{T}$ e $U_{L} \cdots 57$

5.4 .3 - Anālise da variância $\ldots \ldots \ldots \ldots \ldots \ldots$

5.4.4 - Variância da estimativa de um contraste entre duas médias de tratamentos, ajustadas pa

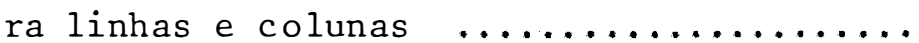

5.5 - Caso de Duas Parcelas Perdidas na Mesma Linha, sendo que Apenas em Uma das Colunas com Parcela Perdida Fi gure o Tratamento Correspondente à Parcela Perdida da Outra Coluna. As Parcelas São Ignoradas ..........

5.5.1 - Sistema de equações normais e estimativas dos parâmetros $\ldots \ldots \ldots \ldots \ldots \ldots \ldots \ldots \ldots . \ldots \ldots$

5.5 .2 - Cálculo das $\mathrm{SQP}_{(1)}, \mathrm{SQP}_{(2)}$ e $\mathrm{SQP}_{(3)} \ldots \ldots \ldots 62$

5.5 .3 - Anālise da variância ...............6 63

5.5.4 - Variância da estimativa de um contraste entre médias de tratamentos, ajustadas para $1 \underline{i}$

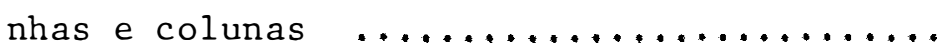


5.6 - Caso de Duas Parcelas Perdidas na Mesma Linha, sendo que Apenas em Uma das Colunas Com Parcela Perdida Fi gure o Tratamento Correspondente à Parcela Perdidada Outra Coluna. As Estimativas das Parcelas Perdidas São Consideradas ....................... 64

5.6.1 - Fórmulas para as estimativas das parcelas per didas ........................... 64

5.6.2 - Förmulas para as correções das somas de quadrados de tratamentos e de linhas: $\mathrm{U}_{\mathrm{T}}$ e $\mathrm{U}_{\mathrm{L}} \cdots \quad 65$

5.6 .3 - Anālise da variância ..............667

5.6.4 - Variância da estimativa de um contraste entre médias de tratamentos ajustadas para linhas e colunas $\ldots \ldots \ldots \ldots \ldots \ldots \ldots \ldots \ldots$

5.7 - Caso de Duas Parcelas Perdidas na Mesma Linha, sendo que em Cada Uma das Colunas Com Parcela Perdida Figu re o Tratamento Correspondente à Parcela Perdida da Outra Coluna e Vice-Versa. As Parcelas São Ignoradas

5.7.1 - Sistema de equações normais e estimativas dos parâmetros $\ldots \ldots \ldots \ldots \ldots \ldots \ldots \ldots \ldots . \ldots 6$

5.7 .2 - Cảlculo das $\mathrm{SQP}_{(1)}, \mathrm{SQP}_{(2)}$ e $\mathrm{SQP}_{(3)} \ldots \ldots \ldots 696$ 5.7 .3 - Anālise da variância $\ldots \ldots \ldots \ldots \ldots \ldots \ldots$

5.7.4 - Variância da estimativa de um contraste entre médias de tratamentos, ajustadas para 1 i nhas e colunas $\ldots \ldots \ldots \ldots \ldots \ldots \ldots \ldots \ldots \ldots$

5.8 - Caso de Duas Parcelas Perdidas na Mesma Linha, sendo que em Cada Uma das Colunas Com Parcela Perdida Figu re o Tratamento Correspondente à Parcela Perdida da Outra Coluna e Vice-Versa. As Estimativas das Parcelas Perdidas São Consideradas 
viii.

Pāg •

5.8.1 - Fórmulas para as estimativas das parcelas per

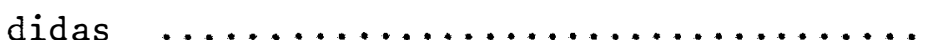

5.8.2 - Fórmulas para as correções das somas de quadrados de tratamentos e de linhas: $\mathrm{U}_{\mathrm{T}}$ e $\mathrm{U}_{\mathrm{L}} \cdots 72$

5.8 .3 - Anālise da variāncia .............. 74

5.8.4 - Variância da estimativa de um contraste entre médias de tratamentos ajustadas para $1 i-$ nhas e colunas

5.9 - Exemplos Numéricos

5.9 .1 - Caso de uma parcela perdida $\left(y_{114}\right) \ldots \ldots \ldots$

5.9.2 - Caso de duas parcelas perdidas na mesma 1inha sendo que o tratamento correspondente à parcela perdida de uma coluna não figure den tre as parcelas remanescentes da outra coluna em que ocorreu a perda e vice-versa $\left(\mathrm{y}_{117}\right.$ e $\left.y_{132}\right)$

5.9.3 - Caso de duas parcelas perdidas na mesma 1inha, sendo que apenas em uma das colunas com parcela perdida figure o tratamento correspondente à parcela da outra coluna $\left(_{114} \mathrm{e}\right.$

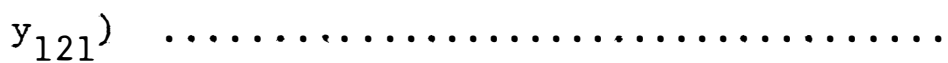

5.9.4 - Caso de duas parcelas perdidas na mesma 1inha, sendo que em cada uma das colunas com parcela perdida figure o tratamento correspondente à parcela perdida da outra coluna e vice-versa $\left(y_{114}\right.$ e $\left.y_{143}\right) \quad \ldots \ldots \ldots \ldots \ldots \ldots$ 
ix.

Pàg.

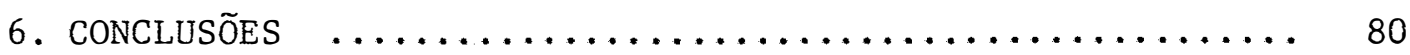

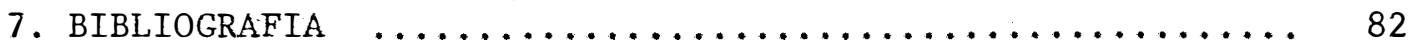




\title{
ESTUDO SOBRE PARCELAS PERDIDAS NO DELINEAMENTO EM QUADRADO DE YOUDEN
}

\author{
Autora: Marinéia de Lara Haddad \\ Orientador: Prof. Dr. Izaias Rangel Nogueira
}

\section{$R E S \cup M O$}

0 presente trabalho teve como objetivo principal a de dução da análise estatística do delineamento em Quadrado de Youden com perda de uma e de duas parcelas na mesma linha. 0 modelo matemá tico considerado foi:

$$
y_{k j i}=m+l_{k}+c_{j}+t_{i(j, k)}+e_{k j i} \text {, }
$$

ou, admitindo-se que os efeitos de colunas incluem a média geral teó rica, e usando-se a forma matricial,

$$
\mathrm{Y}=\mathrm{X} \beta+\varepsilon
$$

Através do método dos quadrados mínimos foram determinadas as estimativas dos parâmetros e as suas respectivas somas de quadrados.

Eliminando-se do modelo matemático original os efeitos de tratamentos e, posteriormente, os efeitos de linhas, novas 
somas de quadrados dos parâmetros foram determinadas. Das diferenças entre elas determinaram-se as corretas somas de quadrados de tra tamentos e de linhas.

Tornando mínima a soma de quadrados do resíduo, $\mathrm{SQR}$, deduziram-se fórmulas para as estimativas das parcelas perdidas.

Através do método do resíduo condicional determinaram-se as expressões das correçōes das somas de quadrados de tratamentos, SQT, e de linhas, SQL.

Verificou-se que a $S Q R$ está corretamente estimada, pois a estimativa da parcela perdida oferece uma contribuição nula para essa soma de quadrados. Assim sendo, e levando-se em considera ção que a SQT foi ajustada para linhas e colunas e a SQL foi ajustạ da para colunas, concluiu-se que a correta soma de quadrados de colunas è a usual.

Foram obtidas também a matriz de dispersão das estimativas dos parâmetros e a fórmula para a variância da estimativa de um contraste entre duas médias de tratamentos ajustadas.

Embora não apresentado o estudo sobre os componentes de variância das diversas causas de variação, determinou-se que a $\mathrm{SQR}$ perde tantos graus de liberdade quantas forem as parcelas perdi das. 
xii.

\section{MISSING PLOTS IN THE YOUDEN SQUARE DESIGN}

Author:

Adviser:

\author{
Marinêia de Lara Haddad \\ Prof. Dr. Izaias Rangel Nogueira
}

\section{SUMMARY}

The objective of this study was concerned with the development of a statistical analysis for the Youden Square Design with one and two missing plots in the same row. Mathematical model used was:

$$
Y_{k j i}=m+l_{k}+c_{j}+t_{i(j, k)}+e_{k j i}
$$

wich in matrix notation, assuming that column effects includes the mean, could be represented by:

$$
Y=X \beta+\varepsilon \quad
$$

Estimation of parameters and sum of squares were done by least squares. Delecting treatment effects from the original model and row effects two new sum of squares of parameters were determined. 
xiii.

By subctration it was calculated correct values for treatment sum of squares and row sum of squares.

By minimization of residual sum of squares formulas were developed for estimation of missing plots as well as correct treatment and row sums of squares. Since the treatment sum of squares was adjusted for rows and columns effects and row sum of squares was adjusted for column it was shown that column sum of squares is the usual.

It was also determined the matrix dispersion of the parameters estimates and the variance for the contrast between two adjusted treatment means. Degrees of freedom lost by the residual sum of squares was equal to the number of missing plots. 


\section{INTRODUÇÃO}

Nos delineamentos em quadrado latino, os tratamentos são dispostos em linhas e colunas, de tal forma, que cada linha ou cada coluna constitui um bloco completo.

Uma estrutura que apresenta uma certa similaridade com os quadrados latinos é aquela em que cada linha constitui um blo co completo, mas as colunas estão estruturadas segundo Blocos Incom pletos Equilibrados.

Esta estrútura constitui os denominados "Quadrados de Youden", nos quais cada tratamento aparece uma vez em cada linha, e cada par de tratamentos aparece nas colunas um mesmo número de vezes.

A omissão de linhas ou de colunas em um quadrado latino pode originar um delineamento em Quadrado de Youden. 
Sabe-se que em trabalhos experimentais è comum ocorrer perdas de parcelas devido a causas acidentais, normalmente alheias ao experimentador. A análise estatística de tais experimentos se torna mais complexa e è há muito tempo estudada.

A determinação de valores estimados para as parcelas perdidas è a solução mais usada para resolver estes casos. Uma vez estimadas as parcelas, procede-se à añálise estatística da maneira usual.

Deve-se salientar que este método citado acima traz um vício que, conforme o caso, pode prejudicar os resultados finais, sendo necessário proceder-se a uma correção das somas de quadrados viciadas .

Em delineamentos em Quadrado de Youden é pouco conhe cido o estudo de parcela perdida, e ainda desconhecido o estudo de parcelas perdidas.

Para tanto, determinaram-se fórmulas para a estimação de uma e de duas parcelas perdidas na mesma linha.

Determinaram-se também as correções das somas de qua drados e as variâncias entre médias de tratamentos ajustadas para linhas e para colunas.

Tambēm faz parte do objetivo deste trabalho a anālise estatistica de um delineamento em Quadrado de Youden com :uma e duas parcelas perdidas na mesma linha, ignorando-se as suas estimativas. 
Os demais casos de perda de parcelas no delineamento em Quadrado de Youden não foram citados devido a grande dificuldade eIn se agrupar as particularidades de cada um deles. 
4.

\section{REVISAOO DE LITERATURA}

Nas anālises estatísticas de experimentos onde ocorrem perdas de parcelas, a grande preocupação inicial é a determinação, sob um aspecto matemático rigoroso, de estimativas para essas observações perdidas.

ALLAN e WISHART (1930). foram os primeiros autores a se preocuparem com a determinação de valores para parcelas perdidas, e a conceituaram como sendo observações imperfeitas, devidas a causas além do controle do próprio experimentador.

Esses autores apresentaram fórmulas para a estimativa de parcela perdida em delineamentos em blocos ao acaso e em quadrado latino.

YATES (1933), substituindo os dados perdidos por incógnitas, e baseado no método dos quadrados mỉnimos, procurou minimizar a soma de quadrados do resíduo. 
Ao citar um experimento em blocos casualizados com uma parcela perdida, o autor afirmou que, se o valor estimado for inserido no lugar da parcela perdida, e se a análise for feita como se nãc tivesse ocorrido nenhuma perda, algumas importanter propriedades passam a existir:

a) As estimativas dos efeitos de tratamentos e blocos s são as mesmas que as obtidas pelo correto método dos quadrados mínimos.

b) A soma de quadrados do resíduo é exatamente a mesma que a dada pelo procedimento correto.

c) 0 número de graus de liberdade associado ao total e ao resí duo fica reduzido de uma unidade.

Mostrou ainda o autor que a soma de quadrados de tra tamentos, levando-se em consideração o valor estimado da unidade per dida, està sempre superestimada.

0 mesmo autor apresentou, para delineamentos em blocos casualizados e em quadrado 1 atino, fórmulas para a variância de um contraste de médias de dois tratamentos, sendo que em um dos tra tamentos figura a parcela perdida.

ANDERSON (1946) definiu parcela perdida de maneira análoga a ALLAN e WISHART (1930), e apresentou uma completa revisão de literatura sobre o assunto.

Apresentou ainda determinações de fórmulas para esti mativas de unidades perdidas em värios tipos de delineamentos: blocos ao acaso, blocos ao acaso com a aplicação dos esquemas fatoriais $2^{\mathrm{n}}$ e $3^{\mathrm{n}}$, quadrado latino, lâtice e parcelas subdivididas. 
o referido autor aplicou o método da covariância em um experimento em parcelas subdivididas com $r$ repetições, $\alpha$ parcelas e B subparcelas, onde a observação perdida recebeu os tratamentos $a_{1}, b_{1}$, na repetição $r_{1}$, e estabeleceu como a melhor estimativa da subparcela perdida, a fim de minimizar o erro da mesma, o resíduo do coeficiente $\underline{b}$ de regressão:

$$
y=\frac{r\left(R_{1} A_{1}\right)+B\left(A_{1} B_{1}\right)-A_{1}}{(r-1)(\beta-1)},
$$

onde, $\mathrm{y}=$ subparcela perdida;

$$
\begin{aligned}
A_{1}= & \text { total de todas as unidades com o tratamento } a_{1} ; \\
B_{1}= & \text { total de todas as unidades com o tratamento } b_{1} ; \\
\left(R_{1} A_{1}\right)= & \text { total de todas as unidades existentes com o tratamento } a_{1} \\
& \text { na repetição } r_{1} ; \\
\left(A_{1} B_{1}\right)= & \text { total de todas as unidades existentes com os tratamentos } \\
& a_{1} \text { e } b_{1} .
\end{aligned}
$$

Afirmou ainda que se esta estimativa for usada para a subparcela perdida, a soma de quadrados do resíduo (b) estará cor retamente estimada, porém as demais estarão ligeiramente superestimadas.

COCHRAN e COX (1957) afirmaram que, quando algumas ob servações estão faltando, o procedimento correto é admitir que todas as observações presentes seguem o modelo matemático proposto e, pelo método dos quadrados mínimos, determinar as equações normais. o problema está na resolução desse sistema de equações normais, o 
qual não apresenta a mesma simetria encontrada quando todas as observações estão presentes.

Assim, os autores adotaram o seguinte método para analisar um experimento com unidades perdidas.

Ap̃os "estimar" os valores das unidades perdidas, inseri-las no quadro de dados e proceder-se à anālise estatística da maneira usual. As somas de quadrados devem ser corrigidas, quando necessário, através de métodos especiais, possibilitando, assim, a aplicação do exato teste $\mathrm{F}$.

Esses autores ainda apresentaram förmulas para o cāl culo de parcelas perdidas em vários tipos de delineamentos.

Para os quadrados latinos incompletos, os autores de terminaram, através da minimização da soma de quadrados do resíduo da análise intrablocos, a estimativa de uma parcela perdida:

$$
x=\frac{\lambda[r C+t B+(t-1) T-G]-r T^{\prime}-(r-1) B_{t}+B_{t^{\prime}}}{r(r-1)(r-2)},
$$

onde: $\mathrm{x}=$ parcela perdida;

$r=$ número de repetições dos tratamentos;

$t=$ nümero de tratamentos;

$\lambda=$ número de vezes que um par de tratamentos aparece na mesma linha (bloco incompleto);

$C$, $B$ e $\mathrm{T}=$ totais da coluna (bloco completo), linha e tratamento que contêm a parcela perdida;

$G=$ total das parcelas disponíveis; 


$$
\begin{aligned}
T^{\prime}= & \text { total de todos os tratamentos que aparecem na linha que } \\
& \text { contém a observação perdida; } \\
B_{t}= & \text { totais das linhas que apresentam o tratamento correspon- } \\
& \text { dente ao da parcela perdida; } \\
B_{t^{\prime}=} & \text { total dos valores } B_{t} \text { para os outros tratamentos que apare } \\
& \text { cem na linha que contêm a parcela perdida. }
\end{aligned}
$$

Se o experimento for repetido $\underline{p}$ vezes:

$$
x=\frac{p\left[p k \lambda C+p t \lambda B+k(k-1) T-p \lambda R-k T^{\prime}-(k-1) B_{t}+B_{t^{\prime}}\right]}{r(k-1)(p k-p-1)}
$$

onde: $\mathrm{k}=$ número de colunas;

$\mathrm{R}=$ total da repetição em que aparece a observação perdida.

$$
\text { PANSE e SUKHATME (1957), baseados em YATES (1933), }
$$
mostraram um método prätico para estimar duas parcelas perdidas nos delineamentos em blocos casualizados e em quadrado latino.

STEEL e TORRIE (1960) sugeriram que a anālise de variância em experimentos em parcelas subdivididas com unidades perdi das deva ser feita após a introdução das estimativas dos valores das unidades perdidas. Um grau de liberdade deve ser subtraído do erro (b) para cada subparcela perdida do experimento. A estimativa do quadrado médio para o erro (b) é imparcial, sendo que o mesmo não acontece com os demais quadrados médios- Se somente-poucas-observa-. ções forem perdidas, as tendências podem ser ignoradas, ou seja, as correções para as somas de quadrados podem ser desprezadas. 
CAMPOS (1964) apresentou um completo estudo sobre a análise de experimentos com parcelas perdidas nos delineamentos em blocos casualizados, em quadrados latinos e em períodos sucessivos. 0 autor mostrou duas maneiras de se calcular os valores das parcelas perdidas: o método geral de estimação de parcelas perdidas e o método iterativo sob o ponto de vista de sucessões. Ainda salientou o autor que o mais acertado é analisar o experimento com os dados restantes, ignorando-se a perda ocorrida.

PINHO (1973) estudou um experimento em parcelas subdivididas com $\mathrm{J}$ blocos, I parcelas e K subparcelas, e determinou, atravēs da minimização da soma de quadrados do resíduo (b), fórmulas para as estimativas de uma e de duas subparcelas perdidas, com a restrição $k>2$.

Para o caso de uma subparcela perdida, a autora apre sentou as corretas somas de quadrados, determinadas por dois métodos:

- calculadas diretamente através de matrizes;

- corrigidas através de fórmulas deduzidas pelo método do resí duo condicional.

Para o caso de duas subparcelas perdidas, a autora afirmou que o melhor é determinar as somas de quadrados diretamente através de matrizes.

PINHO (1975) apresentou um estudo sobre os componentes de variância das somas de quadrados das diversas causas de variação de um delineamento em parcelas subdivididas com uma subparce la perdida. 
PIMENTEL GOMES (1978) estudou observações perdidas em vários tipos de delineamentos, apresentando fórmulas para suas estimativas e para as correções das somas de quadrados tendenciosas. Salientou que as correções são geralmente desprezíveis, porém, pode-se usar o método do resíduo condicional para se obterem os valores exatos das somas de quadrados das causas de variação.

HADDAD (1978) apresentou um estudo sobre parcela per dida em delineamentos em parcelas subdividas. Determinou, através da minimização da soma de quadrados do resíduo (a) e da soma de qua drados do resíduo (b), fórmulas para as estimativas das subparcelas que compõem a parcela perdida. Através do método do resíduo condicional, a autora determinou a fórmula para a correção da soma de quadrados dos tratamentos principais, demonstrando ainda que a soma de quadrados dos tratamentos secundārios é a usual.

SANCHES (1978) estudou perdas de parcelas, linhas e colunas em um delineamento em quadrado latino, deduzindo fórmulas pa ra as somas de quadrados ajustadas e para as correções das somas de quadrados.

RIBEIRO (1980) deduziu fórmulas para estimativas de uma e de duas subparcelas perdidas, e para correções das somas de quadrados viciadas em delineamento inteiramente casualizadas com par celas subdivididas.

SANCHES (1980) apresentou, para delineamentos em blo cos ao acaso com parcelas subdivididas, fórmulas para estimativas 
de uma e de duas subparcelas ou parcelas perdidas. Demonstrou, ain-

da, fórmulas para se obterem as somas de quadrados ajustadas das di versas causas de variação. 
12.

\section{MATERIAL}

Para comparação dos resultados utilizar-se-ão dados de dois experimentos em Quadrado de Youden, os quais estão apresentados nas Tabelas 1 e 2 .

Os dados da Tabela 1 foram compilados de PIMENTEL GOMES (1978), relativos a um ensaio de competição de variedades de cana-de-açúcar, em quadrado latino $5 \times 5$, onde consideraram-se apenas as quatro primeiras linhas.

A Tabela 2 é composta por dados fictícios. 
Tabela 1 - Produções de cana-planta, em quilos por parcela, onde $A=$ $\mathrm{C0290}, \mathrm{B}=\mathrm{C} 0421, \mathrm{C}=\mathrm{C} 0419, \mathrm{D}=\mathrm{P} 05-2878$ e $\mathrm{E}=\mathrm{CP} 36-13$ são as variedades de cana.

\begin{tabular}{|c|c|c|c|c|c|c|}
\hline & & & & & & $\begin{array}{l}\text { TOTAIS DE } \\
\text { LINHAS }\end{array}$ \\
\hline & $\mathrm{D}$ & A & B & $C$ & $E$ & \multirow{3}{*}{2.322} \\
\hline & 432 & 518 & 458 & 583 & 331 & \\
\hline & C & $E$ & A & B & $\mathrm{D}$ & \\
\hline & 724 & 478 & 524 & 550 & 400 & \multirow[t]{3}{*}{2.676} \\
\hline & $\mathrm{E}$ & B & $C$ & D & A & \\
\hline & 489 & 384 & 556 & 297 & 420 & \\
\hline & B & D & $E$ & A & C & \\
\hline & 494 & 500 & 313 & 486 & 501 & 2.294 \\
\hline $\begin{array}{l}\text { TOTAIS DE } \\
\text { COLUNAS }\end{array}$ & 2.139 & 1.880 & 1.851 & 1.916 & 1.652 & 9.438 \\
\hline
\end{tabular}

Tabela 2 - Rendimentos de tomate, em kg/parcela, onde A, B, C, D, E, F e $G$ sào tipos de inseticidas (dados fictícios).

\begin{tabular}{|c|c|c|c|c|c|c|c|c|}
\hline \multirow{2}{*}{ LINHAS } & \multicolumn{7}{|c|}{$C O L U N A S$} & \multirow{2}{*}{$\begin{array}{c}\text { TOTAIS } \\
\text { DE } \\
\text { LINHAS }\end{array}$} \\
\hline & 1 & 2 & 3 & 4 & 5 & 6 & 7 & \\
\hline \multirow{2}{*}{ I } & $G$ & $\mathrm{~A}$ & B & C & $\mathrm{D}$ & & & \multirow{6}{*}{$\begin{array}{l}238,60 \\
201,80\end{array}$} \\
\hline & $\quad 22,7$ & 32,6 & 37,6 & 37,2 & 34,5 & 44,4 & 33,6 & \\
\hline \multirow{2}{*}{ II } & A & B & $\mathrm{C}$ & D & $\mathrm{E}$ & $\mathrm{F}$ & G & \\
\hline & 18,1 & 26,8 & 32,2 & 41,2 & 26,8 & 33,1 & 23,6 & \\
\hline \multirow{2}{*}{ III } & $\mathrm{C}$ & $\mathrm{D}$ & $E$ & $\mathrm{~F}$ & G & $\mathrm{A}$ & B & \\
\hline & 19,5 & 25,9 & 26,3 & 44,5 & 24,5 & 32,2 & 23,0 & \\
\hline $\begin{array}{l}\text { TOTAIS DE } \\
\text { COLUNAS }\end{array}$ & 60,3 & 85,3 & 96,1 & 122,9 & 85,8 & 105,7 & 80,2 & 636,30 \\
\hline
\end{tabular}


Os parâmetros que caracterizam os delineamentos em quadrados de Youden são representados, neste trabalho, por:

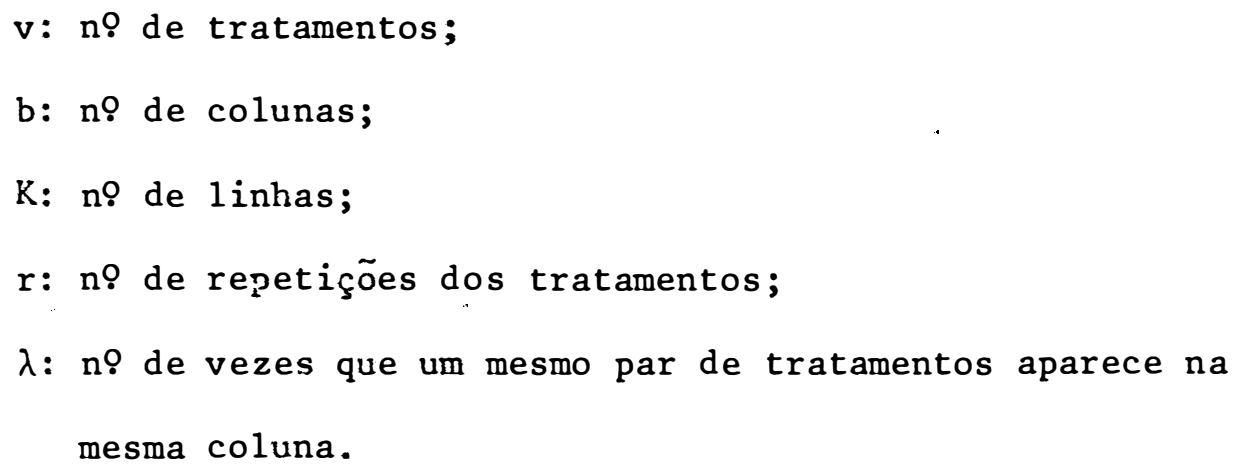

Propriedades:

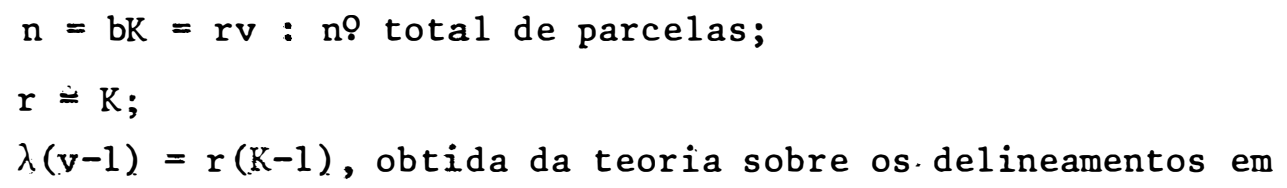


15.

\section{1 - Modelo Matemātico}

Cada parcela de um experimento em Quadrado de Youden é regida pelo modelo matemático

$(4.1 . a)$

$$
y_{k j i}=m+l_{k}+c_{j}+t_{i(j, k)}+e_{k j i},
$$

onde:

$$
\begin{aligned}
& y_{k j i}=\text { observação do } i \text {-ésimo tratamento na k-ésima linha e } \\
& \mathrm{m}=\text { média geral } \\
& \ell_{k}=\text { efeito da } k \text {-ésima linha }(k=1,2, \ldots, k) \text {; } \\
& c_{j}=\text { efeito da } j \text {-ésima coluna }(j=1,2, \ldots, b) \text {; } \\
& t_{i(j, k)}=\text { efeito do } i \text {-ésimo tratamento }(i=1,2, \ldots, v) \text {; } \\
& e_{k j i}=\text { erro experimental, onde se supõe } e_{k j i} \cap N\left(0, \sigma^{2}\right) \text {. } \\
& \text { Admitindo-se que os efeitos de colunas incluam a mé- } \\
& \text { dia geral teörica, e usando-se a forma matricial, o modelo (4.1.a) }
\end{aligned}
$$

fica:

$$
\text { (4.1.b) } \quad \mathrm{Y}=\mathrm{XB}+\varepsilon,
$$

onde:

$$
\begin{aligned}
& \mathrm{Y}=\text { vetor dos dados observados; } \\
& \mathrm{X}=\text { matriz da forma }\left[\begin{array}{lll}
\mathrm{X}_{1} & \vdots \mathrm{X}_{2} & \vdots \\
\mathrm{X}_{3}
\end{array}\right] ; \\
& \mathrm{X}_{1}=\text { matriz correspondente aos tratamentos; } \\
& \mathrm{X}_{2}=\text { matriz correspondente às linhas; } \\
& \mathrm{X}_{3}=\text { matriz correspondente às colunas; }
\end{aligned}
$$




$$
\begin{aligned}
& B=\text { vetor da forma }\left[\begin{array}{l}
\tau \\
\ell \\
c
\end{array}\right] ; \\
& \tau=\text { vetor dos efeitos de tratamentos; } \\
& \ell=\text { vetor dos efeitos de linhas; } \\
& c=\text { vetor dos efeitos de colunas; } \\
& \varepsilon=\text { vetor dos erros experimentais, sendo que } \varepsilon \cap N\left(\phi, \sigma^{2} I_{\bar{n}}\right) .
\end{aligned}
$$

\section{2 - Sistema de Equações Normais}

O sistema de equações normais de um delineamento em Quadrado de Youden, obtido através dos métodos dos quadrados minimos, è:

$$
X^{\prime} X \bar{B}=X^{\prime} Y
$$

ou seja,

$(4.2 . b)$

$$
\left[\begin{array}{ccc}
X_{1}^{\prime} X_{1} & X_{1}^{\prime} X_{2} & X_{1}^{\prime} X_{3}^{-} \\
X_{2}^{\prime} X_{1} & X_{2}^{\prime} X_{2} & X_{2}^{\prime} X_{3} \\
X_{3}^{\prime} X_{1} & X_{3}^{\prime} X_{2} & X_{3}^{\prime} X_{3}
\end{array}\right]\left[\begin{array}{c}
\hat{\tau} \\
\bar{l} \\
\hat{c}
\end{array}\right]=\left[\begin{array}{c}
X_{1}^{\prime} Y \\
X_{2}^{\prime} Y \\
X_{3}^{\prime} Y
\end{array}\right]
$$

Para facilitar os desenvolvimentos posteriores,o sis tema de equações normais (4.2.b) é representado por:

(4.2.c) $\quad\left[\begin{array}{ccc}\mathrm{R}_{\mathrm{vv}} & \mathrm{E}_{\mathrm{bK}} & \mathrm{W}_{\mathrm{vb}} \\ \mathrm{E}_{\mathrm{Kb}}^{\prime} & \mathrm{N}_{\mathrm{KK}} & \mathrm{E}_{\mathrm{Kb}}^{\prime} \\ \mathrm{W}_{\mathrm{bv}}^{\prime} & \mathrm{E}_{\mathrm{bK}} & \mathrm{H}_{\mathrm{bb}}\end{array}\right]\left[\begin{array}{c}\hat{\tau} \\ \hat{l} \\ \hat{\mathrm{c}}\end{array}\right]=\left[\begin{array}{c}\mathrm{T} \\ \mathrm{L} \\ \mathrm{C}\end{array}\right]$


onde:

$$
\begin{aligned}
& R_{v v}=X_{1}^{\prime} X_{1}=\operatorname{diag}\left(r_{1}, r_{2}, \ldots, r_{v}\right)=r I_{v} \\
& r_{i}=\text { no de repetiçōes dos tratamentos }(i=1,2 \text {, } \\
& \ldots, v) \text {; } \\
& \mathrm{N}_{k K}=\mathrm{X}_{2}^{\prime} \mathrm{X}_{2}=\operatorname{diag}\left(\mathrm{n}_{1}, \mathrm{n}_{2}, \ldots, \mathrm{n}_{\mathrm{K}}\right)=\mathrm{b} \mathrm{I}_{\mathrm{K}} \\
& \mathrm{n}_{\mathrm{k}}=\text { tamanho da linha } \mathrm{k}(\mathrm{k}=1,2, \ldots, k) \text {; } \\
& \mathrm{H}_{\mathrm{bb}}=\mathrm{x}_{3}^{\prime} \mathrm{x}_{3}=\operatorname{diag}\left(\mathrm{h}_{1}, \mathrm{~h}_{2}, \ldots, \mathrm{h}_{\mathrm{b}}\right)=\mathrm{KI}_{\mathrm{b}} \\
& h_{j}=\text { tamanho da coluna } j(j=1,2, \ldots, b) \text {; } \\
& E_{b K}=X_{1}^{\prime} X_{2}=X_{3}^{\prime} X_{2}=\text { matriz de incidencia dos tratamentos em } \\
& \text { relação às Iinhas; } \\
& h_{v b}=x_{1}^{\prime} x_{3}=\text { matriz de incidência em relação às colunas; } \\
& I=X: Y=\text { vetor dos totais de tratamentos; } \\
& L=X_{2}^{Y}=\text { vetor dos totais de linhas; } \\
& C=X_{3}^{Y} Y=\text { vetor dos totais de colunas. }
\end{aligned}
$$

4.3 - Sistema de Equações Normais para o Caso de Perda de Uma ou de Duas Parcelas na Mesma Linha

$$
\text { O sistema de equações normais de um delineamento em }
$$
Quadrado de Youden, onde houve perda de uma ou de duas parcelas na mesma linha, foi obtido pela mesma metodologia de 4.2 , e é representado por:

$(4.3 . a)$

$\left[\begin{array}{ccc}R_{v v} & E_{b K} & W_{v b} \\ E_{K b}^{\prime} & N_{K K} & B_{k b} \\ W_{b v}^{\prime} & B_{b K}^{\prime} & H_{b b}\end{array}\right]\left[\begin{array}{c}\hat{\tau} \\ \hat{\imath} \\ \hat{c}\end{array}\right]=\left[\begin{array}{c}T \\ L \\ C\end{array}\right]$,


onde:

$$
\begin{aligned}
& R_{V V}=X_{1}^{\prime} X_{1}=\operatorname{diag}\left(r_{1}, r_{2}, \ldots, r_{V}\right) \text {; } \\
& N_{\mathrm{KH}}=\mathrm{X}_{2}^{\prime} \mathrm{X}_{2}=\operatorname{diag}\left(\mathrm{n}_{2}, \mathrm{n}_{2}, \ldots, \mathrm{n}_{\mathrm{k}},\right. \\
& H_{b b}=x_{3}^{\prime} x_{3}=\operatorname{diag}\left(h_{1}, h_{2}, \ldots, h_{b}\right) \text {; } \\
& E_{b K}=X_{3}^{\prime} X_{2}=\text { matriz de incidencia dos tratamentos em relação } \\
& \text { às linhas; } \\
& \mathrm{B}_{\mathrm{NB}}=\mathrm{X}_{2}^{\prime} \mathrm{X}_{3}=\text { matriz de incidencia das linhas em relação às co } \\
& \text { Iunas; } \\
& W_{v b}, T, L, C, r_{i}, n_{k}, h_{j} \text { seguem a mesma definição de } 4.2 . \\
& \text { Para maior facilidade nas demonstraçōes posteriores, }
\end{aligned}
$$

$$
F \bar{B}=X^{\prime} Y
$$

\section{4 - Estimativas dos Efeitos dos Parämetros}

Do sistema de equações normais (4.3.b), tem-se que $F$ é una matriz singular e de caracteristica igual a $(v+b+k-2)$, portan to, admitindo-se conjuntamente as restrições:

$$
\sum \vec{d}_{k}=0 \text { e } \quad \sum \hat{c}_{j}=0 \text {, }
$$

tem-se:

$$
\text { (4.4.a) } \quad F_{1} \bar{B}=X^{\prime} Y^{\prime} \text {, }
$$

onde $F_{1}$ é uma matriz não-singular, e dada por: 


$$
F_{2}=\left[\begin{array}{ccc}
R_{v v} & \left(E_{b k^{\prime}}-A_{b k}\right) & \left(w_{v b}-A_{v b}\right) \\
E_{k b}^{\prime} & N_{k K} & \left(B_{k b}-A_{k b}\right) \\
W_{b v}^{\prime} & \left(B_{b k}^{\prime}-A_{b k}\right) & F_{b b}
\end{array}\right]
$$

onde $A_{b K}$, $A_{v b}$, A kb são matrizes cujos elementos são todos iguais a um.

$$
\text { Assim sendo, obtêm-se as estimativas dos parâmetros: }
$$

$$
\hat{\mathrm{E}}=\mathrm{F}_{1}^{-1} X^{\prime} Y
$$

$$
4.5 \text { - Cálculo das } \operatorname{SQP}\left(\bar{l}_{k}, \bar{c}_{j}, \bar{t}_{i}(j, k), \operatorname{SQP}\left(\bar{d}_{k}, \tilde{c}_{j}\right) \text { e } \operatorname{SQP}\left(\bar{c}_{j}\right)\right.
$$

A partir da solução (4.4.b) determina-se a soma de quadrados dos parâmetros, $\operatorname{sQP}\left(\bar{\chi}_{k}, \bar{c}_{j}, t_{i(j, k)}\right)$, que é denominada SQP(I), dada por:

$$
\operatorname{SQP}(1)=\bar{B}^{\prime} X^{\prime} Y=\hat{\tau}^{\prime} T+\ell^{\prime} L+\hat{C}^{\prime} C .
$$

Eliminando-se do modelo matemätico (4.1.a) os efeitos de tratamentos, tem-se o modelo matemático de um delineamento em blocos ao acaso, dado pur:

$$
y_{k j}=m+\ell_{k}+c_{j}+e_{k j} .
$$

Usando-se a forma matricial, e admitindo-se que os efeitos de colunas incluam a média geral teórica, o modelo (4.5.b) fica:

$$
Y^{*}=X^{*} \beta^{*}+\varepsilon^{*},
$$


ond e :

$$
\begin{aligned}
& \mathrm{Y}^{*}=\text { vetor dos dados observados; } \\
& \mathrm{X}^{*}=\text { matriz da forma }\left[\mathrm{X}_{2} \vdots \mathrm{X}_{3}\right] \text {, onde } \mathrm{X}_{2} \text { e } \mathrm{X}_{3} \text { são dadas em } \\
& \text { 4.1; } \\
& \beta *=\text { vetor da forma }\left[\begin{array}{l}
\ell \\
c
\end{array}\right] \text {, onde } \ell \text { e c são dados em 4.1; } \\
& \varepsilon^{*}=\text { vetor dos erros experimentais, onde } \varepsilon^{*} \cap N\left(\phi, \sigma^{2} I_{n}\right) \text {. }
\end{aligned}
$$
blocos ao acaso, onde houve perda de uma ou de duas parcelas no mes mo bloco, foi obtido pela mesma metodologia de 4.2 , e é representado por:

$$
\left[\begin{array}{c}
N_{K K} \\
B_{b K}^{\prime}
\end{array}\right.
$$$$
\left.\begin{array}{c}
\mathrm{B}_{\mathrm{Kb}} \\
\mathrm{H}_{\mathrm{bb}}
\end{array}\right]
$$$$
\left[\begin{array}{l}
\tilde{x} \\
\tilde{c}
\end{array}\right]=\left[\begin{array}{l}
\mathrm{L} \\
\mathrm{C}
\end{array}\right]
$$

onde o simbolo ( ) é utilizado para diferenciar das estimativas dos parâmetros do delineamento em Quadrado de Youden.

Pelo mesmo motivo discutido em 4.3 , o sistema de equa ções (4.5.d) é representado por:

$$
\mathrm{F} * \widetilde{B} *=X * 1 *
$$

Do sistema acima tem-se que $F^{*}$ é uma matriz singular. Admitindo-se a restrição $\sum \hat{c}_{j}=0$, tem-se o novo sistema:

$$
\mathrm{F} * \tilde{\beta} * \mathrm{X}^{*}{ }^{\prime} \mathrm{Y} *,
$$

onde a matriz $\mathrm{F}_{1}^{*}$, não-singular, é dada por: 


$$
F^{*}=\left[\begin{array}{cc}
N_{\mathrm{KK}} & \left(\mathrm{B}_{\mathrm{Kb}}-\mathrm{A}_{\mathrm{Kb}}\right) \\
\mathrm{B}_{\mathrm{bK}}^{\prime} & \mathrm{H}_{\mathrm{bb}}
\end{array}\right] \text {, }
$$

onde $\mathrm{A}_{\mathrm{Kb}}$ foi definida em 4.4 .

Assim sendo, obtêm-se:

$$
\widetilde{B} *=\left(E_{1}^{*}\right)^{-1} X *^{\prime} Y *
$$

ou seja, as estimativas dos parâmetros $\tilde{l}_{k}$ e $\tilde{c}_{j}$.

A soma de quadrados de parâmetros, $\operatorname{SQP}\left(\tilde{d}_{k}, \tilde{c}_{j}\right)$, deno minada $\operatorname{SQP}(2)$, é:

$$
\operatorname{SQP}(2)=\tilde{B} *^{\prime} X^{*} Y *=l^{\prime} L+\tilde{c}^{\prime} C .
$$

$$
\text { Eliminando-se do modelo matemático (4.1.a) os efei- }
$$
tos de tratamentos e de linhas, tem-se o modelo matemático de um de lineamento inteiramente casualizado, dado por:

$$
\begin{aligned}
& y_{j}=m+c_{j}+e_{j} . \\
& \text { Admitindo-se que os efeitos de colunas incluem a mé- } \\
& Y * *=X * * \beta * \star+\varepsilon * \star,
\end{aligned}
$$
dia geral, matricialmente (4.5.i) è dado por:

onde:

$$
\begin{aligned}
& \mathrm{Y*}=\text { vetor dos dados observados; } \\
& \mathrm{X} * \text { = matriz correspondente às colunas; } \\
& B^{* *}=\text { vetor dos efeitos de '.colunas; } \\
& \varepsilon^{* *}=\text { vetor dos erros experimentais. }
\end{aligned}
$$


O sistema de equações normais para esse caso é:

$$
\mathrm{H}_{\mathrm{bb}} \overline{\mathrm{c}}=\mathrm{C},
$$

onde o símbolo (_) é utilizado para indicar as estimativas dos efei tos de colunas do modelo $(4 \cdot 5 \cdot j)$.

Como jā foi visto, $\mathrm{H}_{\mathrm{bb}}$ é uma matriz diagonal, portan

to tem-se que:
$(4.5 . \mathrm{m})$

$$
\hat{c}=\mathrm{H}_{\mathrm{bb}}^{-1} \mathrm{C}
$$

Assim, a soma de quadrados dos parâmetros, $\operatorname{SQP}\left(\hat{\underline{c}}_{j}\right)$, denominada SQP(3), é

$$
\operatorname{SQP}(3)=\hat{c}^{\prime} \mathrm{C}
$$

4.6 - Estimativas dos Efeitos de Tratamentos $\bar{\tau}$, Através do Siste ma Reduzido

Do sistema de equações normais $(4.3 . a)$, obtêm-se:

$(4.6 . a)$

$(4.6 . b)$

$(4.6 . c)$

$$
\left.\begin{array}{l}
R_{v v} \hat{\tau}+E_{b K} \hat{l}+W_{v b} \hat{c}=T \\
E_{K b}^{\prime} \tau+N_{K K} \hat{l}+B_{K b} \hat{c}=L \\
W_{b v}^{\prime} \hat{\tau}+B_{b K}^{\prime} \bar{l}+H_{b b} \hat{c}=c
\end{array}\right]
$$

Admitindo-se a restrição $\sum_{k} \widehat{l}_{k}=0$, então o produto $\left(B_{b K}^{\prime}\right.$. $\left.\grave{l}\right)$ serā igual a $\left[\left(B_{b K}^{\prime}-A_{b K}\right)\right.$ l] , onde $A_{b K}$ é uma matriz definida em 4.4 . 
Para eliminarem-se os efeitos das estimativas de coIunas das equações $(4.6 . a)$ e (4.6.b), deve-se somar à equação(4.6.a) a equação (4.6.c) pré-multiplicada por $\left(-\mathrm{w}_{v b} \mathrm{H}_{b b}^{-1}\right)$, e à equação (4.6.b) a equação (4.6.c) pré-multiplicada por $\left(-\mathrm{B}_{\mathrm{Kb}} \mathrm{H}_{\mathrm{bb}}^{-1}\right)$.

Assim sendo, obtém-se

$$
\begin{aligned}
\text { (4.6.d }) & \left(R-W^{-1} W^{\prime}\right) \hat{\tau}+\left[E-W^{-1}\left(B^{\prime}-A\right)\right] \bar{l}+\phi \hat{C} & =T-W H^{-1} C \\
(4.6 . e) & \left(E^{\prime}-B^{-1} W^{\prime}\right) \hat{\tau}+\left[N-B H^{-1}\left(B^{\prime}-A\right)\right] l+\phi \hat{C} & =L-B H^{-1} C \\
(4.6 . f) & H^{-1} W^{\prime} \hat{\tau}+H^{-1}\left(B^{\prime}-A\right) l+I_{b} \hat{C} & =H^{-1} C
\end{aligned}
$$

Como a matriz $\left[\mathrm{N}-\mathrm{BH}^{-1}\left(\mathrm{~B}^{\prime}-\mathrm{A}\right)\right]$ é não-singular, en tão, se (4.6.e) for pré-multiplicada por $\left[N-B^{-1}\left(B^{\prime}-A\right)\right]^{-1}$, obtề-se:

$$
\text { (4.6.g) } \begin{aligned}
{\left[\mathrm{N}-\mathrm{BH}^{-1}\left(\mathrm{~B}^{\prime}\right.\right.} & -\mathrm{A})]^{-1}\left[\mathrm{E}^{\prime}-\mathrm{BH}^{-1} \mathrm{~W}^{\prime}\right] \bar{\tau}+ \\
+ & I_{K} \ell+\phi=\left[\mathrm{N}-\mathrm{BH}^{-1}\left(\mathrm{~B}^{\prime}-\mathrm{A}\right)\right\rfloor^{-1}\left[\mathrm{~L}-\mathrm{BH}^{-1} \mathrm{C}\right]
\end{aligned}
$$

Para isolar-se o vetor das estimativas dos parâmetros $\hat{\tau}$, deve-se pré-multiplicar $(4.6 . g)$ por $\left\{-\left[E-\mathrm{WH}^{-1}\left(B^{\prime}-A\right)\right]\right\}$ e somar-se em (4.6.d). Assim sendo, obtém-se o sistema reduzido:

$$
\text { (4.6.h) } \quad \mathrm{P} \hat{\tau}=\mathrm{Q},
$$

onde:

$$
\begin{aligned}
& P=\left[R-\mathrm{WH}^{-1} \mathrm{~W}^{\prime}\right]-\left[E-\mathrm{WH}^{-1}\left(\mathrm{~B}^{\prime}-\mathrm{A}\right)\right]\left[\mathrm{N}-\mathrm{BH}^{-1}\left(\mathrm{~B}^{\prime}-\mathrm{A}\right)\right]^{-1}\left[\mathrm{E}^{\prime}-\mathrm{BH}^{-1} \mathrm{~W}^{\prime}\right] \\
& \mathrm{Q}=\left[\mathrm{T}-\mathrm{WH}^{-1} \mathrm{C}\right]-\left[\mathrm{E}-\mathrm{WH}^{-1}\left(\mathrm{~B}^{\prime}-\mathrm{A}\right)\right]\left[\mathrm{N}-\mathrm{BH}{ }^{-1}\left(\mathrm{~B}^{\prime}-\mathrm{A}\right)\right]^{-1}\left[\mathrm{~L}-\mathrm{BH}^{-1} \mathrm{C}\right]
\end{aligned}
$$


Como a matriz $\mathrm{P}$ tem caracteristica igual a $(v-1)$, se gundo CHAKRABARTI (1962), deve-se introduzir uma restrição que não seja função linear estimável dos parâmetros. Sendo assim, obtém-se um novo sistema de equações:

$$
S \hat{\tau}=\phi
$$

Subtraindo-se $(4.6 . j)$ de $(4.6 . i)$, obtém-se:

$$
[P-S] \widehat{\tau}=Q
$$

Seja $[P-S]=M$, não-singular, logo:

$(4.6 . j)$

$$
\bar{\tau}=M^{-1} Q \quad \text {. }
$$

\section{7 - Estimativa de Uma Parcela Perdida}

A estimativa de uma parcela perdida de um delineamen to em Quadrado de Youden será determinada através do método dos qua drados mínimos, ou seja, através da minimização da soma de quadrados do resíduo ( $\mathrm{SQR}$ ).

Tornando minima essa soma de quadrados, obtém-se uma equação relativa à incögnita $\mathrm{x}$, onde $\mathrm{x}$ representa a parcela perdida do experimento. 


\section{8 - Estimativas de Duas Parcelas Perdidas na Mesma Linha}

Serão abordados três casos distintos:

4.8.1) Caso em que o tratamento correspondente "à parcela perdida de uma coluna não figure dentre as parcelas remanescentes da outra coluna em que ocorreu a perda, e vice-versa.

4.8.2) Caso em que apenas em uma das colunas com parcela perdida figure o tratamento correspondente à parcela perdida da outra coluna.

4.8.3) Caso em que em cada uma das colunas com parcela per dida figure o tratamento correspondente à parcela perdida da outra coluna e vice-versa.

As estimativas de duas parcelas perdidas na mesma li nha são determinadas pela mesma metodologia dada em 4.7.

Minimizando-se a SQR obtêm-se duas equações relativas às incöginitas $x_{1}$ e $x_{2}$.

Verifica-se que esse sistema de equações é determina do, de onde se obtém a solução imediata para as incógnitas $x_{1}$ e $x_{2}$, onde $x_{1}, x_{2}$ representam as parcelas perdidas do experimento. 
4.9 - Correção das Somas de Quadrados, Levando-se em Consideração a Estimativa de Uma Parcela Perdida

Para obtenção das verdadeiras somas de quadrados è utilizado o método do resíduo condicional, que é baseado no método dos quadrados mínimos.

As somas de quadrados são representadas pelos seguin tes termos e expressões:

$$
\operatorname{SQTratamentos}_{(\mathrm{x})}=\mathrm{SQT}_{(\mathrm{x})}=\frac{\mathrm{r}}{\lambda \mathrm{v}} \mathrm{V}^{\prime} \mathrm{V},
$$

a qual corresponde à soma de quadrados de tratamentos ajustados para coluna de um delineamento completo em Quadrado de Youden (CHAKRA BARTI, 1962), onde:

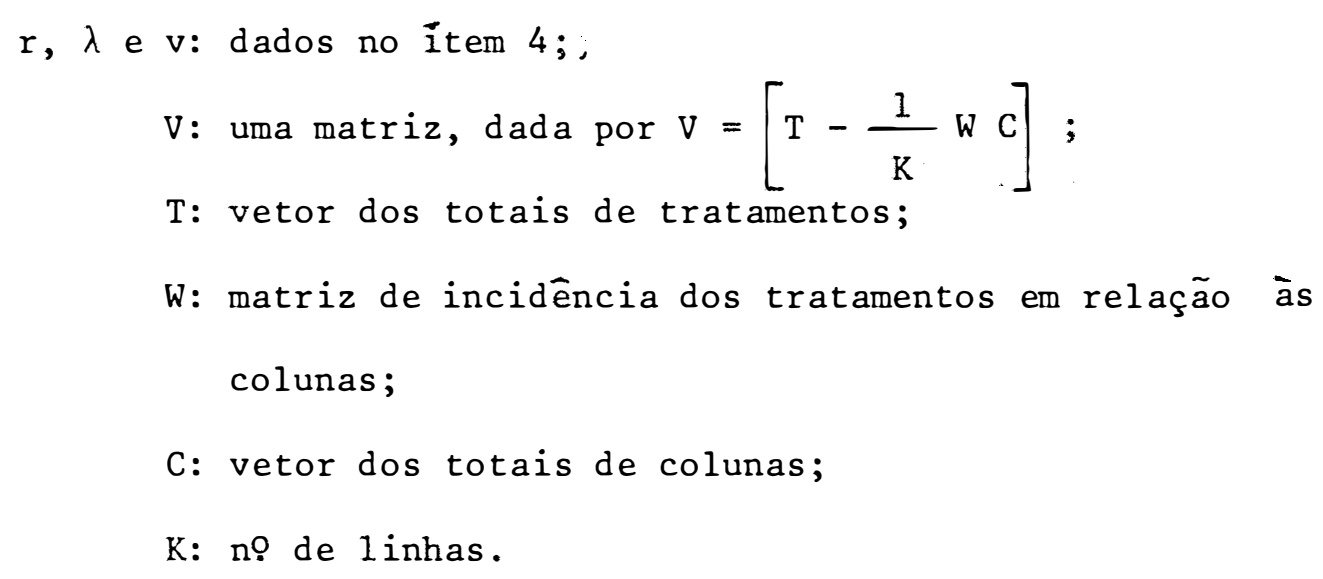

$$
\begin{aligned}
& \text { SQColuna }_{(x)}=S_{(x)}=\sum_{j=1}^{J} \frac{C_{j}^{2}}{K}-\operatorname{Corr}(x) \\
& \text { SQLinha }_{(x)}=S_{(x)}=\sum_{k=1}^{K} \frac{L_{k}^{2}}{b}-\operatorname{Corr}(x)
\end{aligned}
$$




$$
\begin{aligned}
\operatorname{SQTotal}_{(x)}= & \sum_{\mathrm{kji}}^{\prime} \mathrm{y}_{\mathrm{kji}}^{2}-\operatorname{Corr}(\mathrm{x}) \\
\operatorname{SQResiduo}_{(\mathrm{x})}=\mathrm{SQR}_{(\mathrm{x})}=\mathrm{SQTotal}_{(\mathrm{x})} & -\mathrm{SQL}_{(\mathrm{x})}-\mathrm{SQC}_{(\mathrm{x})}- \\
\operatorname{Corr}_{(\mathrm{x})}= & -\frac{\left(\mathrm{GQT}_{(\mathrm{x})}+\mathrm{x}\right)^{2}}{\mathrm{bK}},
\end{aligned}
$$

nde: $G^{*}=$ total geral das parcelas existentes.

$$
\text { Utilizou-se a notação (x) para indicar as somas de }
$$
quadrados que contêm a estimativa da parcela perdida.

$$
\text { Utilizar-se-ão as notações (aj) e (usual) para indi- }
$$
car as somas de quadrados ajustadas e as determinadas ignorando-se a parcela perdida, respectivamente.

A estimativa da parcela perdida oferece uma contribuição nula para a $\mathrm{SQR}$, consequentemente esta está corretamente estimada.

Para a correção da SQT $(x)$ considera-se o delineamento como sendo em blocos ao acaso, onde as linhas são os blocos e as colunas os tratamentos, cujo modelo matemático foi dado em (4.5.b). Para esse caso tem-se:

$$
\mathrm{SQR}_{(y)}=\mathrm{SQTotal}_{(\mathrm{y})}-\mathrm{SQC}(\mathrm{y})-\mathrm{SQL}(\mathrm{y}) \text {, }
$$

onde y representa a parcela perdida quando se considera o delineamen to como sendo em blocos ao acaso.

$$
\begin{aligned}
& \text { Conforme YATES (1933), tem-se: } \\
& \left.\qquad \operatorname{SQT}_{(\mathrm{x})}>\mathrm{SQT}_{(\mathrm{aj}}\right)^{\prime}
\end{aligned}
$$


então existe um nümero real $U$, tal que:

$$
U_{T}=S Q T(x)-S Q T(a j)
$$

Sabe-se também que:

$(4.9 .1)$

$$
\mathrm{SQR}_{(y)}-\mathrm{SQR}_{(\mathrm{x})}=\mathrm{SQT}_{(\mathrm{aj})}
$$

Assim,

$$
\mathrm{SQT}_{(a j)}=\mathrm{SQT}_{(\mathrm{x})}-\mathrm{U}_{\mathrm{t}}
$$

Para a correção da SQL $(x)$ considera-se o delineamento como sendo inteiramente casualizado, onde as colunas são os tratamentos e as linhas as repetições, cujo modelo matemático foi dado em (4.5.i).

$$
\begin{aligned}
& \text { Tem-se, então: } \\
& \mathrm{SQR}_{(z)}=\operatorname{SQTotal}_{(z)}-\mathrm{SQC}_{(z)},
\end{aligned}
$$

onde $z$ representa a parcela perdida para o delineamento inteiramente casualizado.

$$
\text { Sabe-se também que: }
$$

$$
\left.S Q R_{(z)}-S Q R_{(y)}=S Q L_{(a j}\right)
$$

Desenvolvendo-se as somas de quadrados da expressão (4.9.3), tem-se:

$$
\mathrm{SQL}_{(a j)}=\mathrm{SQL}_{(\mathrm{x})}-\mathrm{U}_{\mathrm{L}} \cdot
$$


Como têm-se SQRcorreta, SQL $(a j)$ e SQT $(a j)$, então a SQC só poderá ser a usual, ou seja, SQC (usual)

4.10 - Correção das Somas de Quadrados, Levando-se em Consideração as Estimativas de Duas Parcelas Perdidas na Mesma Linha

As verdadeiras somas de quadrados são obtidas pelo mé todo descrito em 4.9.

As somas de quadrados apresentadas em 4.9 são válidas para 4.10, somente com a diferença de que são acompanhadas da notação $\left(x_{1}, x_{2}\right)$, que indicam a presença das duas estimativas das parcelas perdidas.

$$
\begin{gathered}
\text { Seguindo a mesma metodologia de } 4.9 \text {, tem-se: } \\
\operatorname{SQR}_{\left(\mathrm{y}_{1}, \mathrm{y}_{2}\right)}=\operatorname{SQTotal}_{\left(\mathrm{y}_{1}, \mathrm{y}_{2}\right)}-\mathrm{SQC}_{\left(\mathrm{y}_{1}, \mathrm{y}_{2}\right)}-\mathrm{SQL}_{\left(\mathrm{y}_{1}, \mathrm{y}_{2}\right)},
\end{gathered}
$$

onde $y_{1}, y_{2}$ representam as parcelas perdidas na mesma linha, quando se considera o modelo matemático (4.5.b).

Agora,

$(4.10 .1)$

$$
\mathrm{SQR}_{\left(\mathrm{y}_{1}, \mathrm{y}_{2}\right)}-\mathrm{SQR}_{\left(\mathrm{x}_{1}, \mathrm{x}_{2}\right)}=\mathrm{SQT}_{(\mathrm{aj})} \ldots
$$

Desenvolvendo-se a expressão acima, tem-se:

$(4.10 .2)$

$$
\operatorname{SQT}_{(a j)}=\operatorname{SQT}_{\left(x_{1}, x_{2}\right)}-U_{T} \quad .
$$

Ainda seguindo a mesma metodologia de 4.9, tem-se:

$$
\operatorname{SQR}_{\left(z_{1}, z_{2}\right)}=\operatorname{SQTotal}_{\left(z_{1}, z_{2}\right)}-\operatorname{SQC}_{\left(z_{1}, z_{2}\right)} \text {, }
$$


onde $z_{1}, z_{2}$ representam as parcelas perdidas na mesma linha, quando se considera o modelo matemático (4.5.i).

$(4.10 .3)$

$$
\operatorname{SQR}_{\left(z_{1}, z_{2}\right)}-\mathrm{SQR}_{\left(y_{1}, y_{2}\right)}=\mathrm{SQL}_{(\mathrm{aj})}
$$

Desenvolvendo-se as somas de quadrados da expressão

$(4.10 .3)$, tem-se:

$(4.10 .4)$

$$
\mathrm{SQL}_{(a j)}=\mathrm{SQL}_{\left(\mathrm{x}_{1}, \mathrm{x}_{2}\right)}-\mathrm{U}_{\mathrm{L}}
$$

Como jä descrito em 4.9, a SQC correta é também a usual. Todas as correções das somas de quadrados mencionadas em 4.10 são válidas para todos os casos descritos em 4.8, ou seja, (4.8.1), $(4.8 .2)$ e $(4.8 .3)$.

\subsection{1 - Verdadeiras Somas de Quadrados}

\subsection{1 - Soma de quadrados de tratamentos, ajustada para linhas e colunas}

Um dos métodos de obtenção da soma de quadrados de tratamentos ajustada para linhas e colunas, $S Q T(a j)$ ou $S Q(T \mid \ell, C), e ́$ através da diferença entre a $\mathrm{SQP}_{(1)}$ e a $\mathrm{SQP}_{(2)}$, dadas em (4.5.a) e $(4.5 . h)$, respectivamente, ou seja:
$(4.11 .1 . a)$

$$
\mathrm{SQT}_{(a j)}=\mathrm{SQP}_{(1)}-\mathrm{SQP}_{(2)}
$$

A SQT $_{(a j)}$ pode ser também determinada através da solução do sistema reduzido, dado em $(4.6 . j)$, ou seja: 


$$
\widehat{\tau}=M^{-1} Q \quad .
$$

$$
\text { E, portanto: }
$$

$(4.11 .1 . b)$

$$
\operatorname{SQT}_{(a j)}=\tilde{\tau}^{\prime} Q=Q^{\prime}\left(M^{-1}\right)^{\prime} Q \quad .
$$

Levando-se em consideração a estimativa ou estimati vas das parcelas perdidas, a SQT $(a j)$ é determinada como dado em $(4.9 .2)$ e $(4.10 .2)$, respectivamente.

\subsection{2 - Soma de quadrados de linhas ajustada para colu- nas}

A soma de quadrados de linhas ajustada para colunas, $\mathrm{SQL}_{(\mathrm{aj})}$ ou $\mathrm{SQ}(\mathrm{L} \mid \mathrm{C})$, pode ser determinada através da diferença entre a $\operatorname{SQP}_{(2)}$ e a $\mathrm{SQP}_{(3)}$, dadas em $(4.5 . h)$ e $(4.5 . \mathrm{n})$, respectivamente.Assim, $(4.11 .2 . a)$

$$
\mathrm{SQL}_{(\mathrm{aj})}=\mathrm{SQP}_{(2)}-\mathrm{SQP}_{(3)} .
$$

Do sistema de equações normais (4.5.d) tem-se:

$(4.11 .2 . b)$

$$
\left.\begin{array}{l}
\mathrm{N}_{\mathrm{KK}} \tilde{l}+\mathrm{B}_{\mathrm{kb}} \tilde{c}=\mathrm{L} \\
\mathrm{B}_{\mathrm{bK}}^{\prime} \tilde{l}+\mathrm{H}_{\mathrm{bb}} \tilde{c}=\mathrm{C}
\end{array}\right\}
$$

$(4.11 .2 . c)$

Considerando a restrição $\sum_{k=1}^{k} \chi_{k}=0$, então o produto $\left(B_{b K}^{\prime} \tilde{l}\right) \bar{e}$ igual a $\left(B_{b K}^{\prime}-A_{b K}\right) \tilde{l}$. Se a equação $(4.11 .2 . c)$ for pré-multiplicada por $\left(-\mathrm{B} \mathrm{H}^{-1}\right)$ e somada à. equação (4.11.2.b), obtêm-se as estimativas: 
$(4.11 .2 . d)$

$$
\begin{aligned}
& \tau=\left[\mathrm{N}-\mathrm{BH}^{-1}\left(B^{\prime}-A\right)\right]^{-1}\left[L-B H^{-1} C\right] \\
& \tilde{c}=H^{-1} C-\left[H^{-1}\left(B^{\prime}-A\right)\right] \tilde{l}
\end{aligned}
$$

$(4.11 .2 . e)$

Então, (4.5.h) ë dada por:

$$
\begin{aligned}
\mathrm{SQP}_{(2)=} & {\left[\mathrm{L}-\mathrm{BH}^{-1} \mathrm{C}\right]^{\prime}\left\{\left[\mathrm{N}-\mathrm{BH}^{-1}\left(\mathrm{~B}^{\prime}-\mathrm{A}\right)\right]^{-1}\right\}^{\prime} \mathrm{L}+\mathrm{C}^{\prime}\left(\mathrm{H}^{-1}\right)^{\prime} \mathrm{C}-} \\
& -\left[\mathrm{L}-\mathrm{BH}^{-1} \mathrm{C}^{\prime}\right]^{\prime}\left\{\left[\mathrm{N}-\mathrm{BH}^{-1}\left(\mathrm{~B}^{\prime}-\mathrm{A}\right)\right]^{-1}\right\}^{\prime}\left[\mathrm{H}^{-1}\left(\mathrm{~B}^{\prime}-\mathrm{A}\right)\right]^{\prime} \mathrm{C}
\end{aligned}
$$

ou, reagrupando-se e simplificando-se:

$$
\begin{aligned}
& (4.11 .2 . f) \quad S Q P(2)=C^{\prime} H^{-1} C+\left[L-B H^{-1} C\right] \cdot\left\{\left[N-B^{-1}\left(B^{\prime}-A\right)\right]^{-1}\right\}^{\prime} \times \\
& \times\left[L-\left(B^{\prime}-A\right)^{\prime} H^{-1} C\right]
\end{aligned}
$$

Do sistema (4.5.e), tem-se:

$$
\underline{\hat{c}}=\mathrm{H}^{-1} \mathrm{C} \text {. }
$$

Assim, (4.5.n) è dado por:

$(4.11 .2 .8)$

$$
S Q P(3)=C^{\prime} H^{-1} \mathrm{C} \text {. }
$$

Substituindo-se em (4.11.2.a) as expressões (4.11.2.g)

e (4.11.2.g), obtém-se:

$$
\operatorname{SQL}_{(a j)}=\left[L-B H^{-1} C\right]^{\prime}\left\{\left[N-B H^{-1}\left(B^{\prime}-A\right)\right]^{-1}\right\}^{\prime}\left[L-\left(B^{\prime}-A\right)^{\prime} H^{-1} C\right]
$$

Como a expressão acima é uma matriz de dimensão ( $1 \times 1$ ), tem-se:

$$
\begin{aligned}
&(4.11 .2 . h) \quad \text { SQL } \\
&(a j)= {\left[L-\left(B^{\prime}-A\right)^{\prime} H^{-1} C\right]^{\prime}\left[N-B H^{-1}\left(B^{\prime}-A\right)\right]^{-1} \times } \\
& \times\left[L-B H^{-1} C\right]
\end{aligned}
$$


Levando-se em consideração a estimativa ou estimativas das parcelas perdidas, a obtenção da SQL $(a j)$ é dada em (4.9.4) e (4. 0.4$)$, respectivamente.

4.11.3 - Soma de quadrados de colunas

Como a soma de quadrados de tratamentos foi ajustada para linhas e colunas, e a soma de quadrados de linhas foi ajustada para colunas, a soma de quadrados de colunas é a usual.

\subsection{4 - Soma de quadrados do residuo}

$$
\begin{aligned}
& \text { Como visto em }(4.5 . a) \text {, a } \mathrm{SQP}_{(1)} \text { é dada por: } \\
& \mathrm{SQP}_{(1)}=\hat{\tau}^{\prime} \mathrm{T}+\bar{l}^{\prime} \mathrm{L}+\hat{c}^{\prime} \mathrm{C} . \\
& \text { Das equações }(4.6 . e) \text { e }(4.6 . f) \text { obtêm-se, respectiva- }
\end{aligned}
$$

mente que:

$(4.11 .4 . a)$

$$
\begin{aligned}
& d=\left[N-B^{-1}\left(B^{\prime}-A\right)\right]^{-1}\left[L-B H^{-1} C\right]- \\
& -\left[N-B H^{-1}\left(B^{\prime}-A\right)\right]^{-1}\left[E^{\prime}-B H^{-1} W^{\prime}\right] \hat{\tau} \\
& \text { (4.11.4.b) } \quad \bar{C}=\mathrm{H}^{-1} \mathrm{C}-\mathrm{H}^{-1}\left(\mathrm{~B}^{\prime}-\mathrm{A}\right) \bar{l}-\mathrm{H}^{-1} \mathrm{~W}^{1} \hat{\mathrm{T}}
\end{aligned}
$$

Substituindo-se (4.11.4.a) e (4.11.4.b) em (4.5.a), e fazendo-se as devidas simplificações, obtēm-se:

$$
\begin{aligned}
(4.11 .4 . c) \mathrm{SQP}_{(I)=} & \hat{\tau}^{\prime} \mathrm{Q}+\mathrm{C}^{\prime} \mathrm{H}^{-1} \mathrm{C}+ \\
& +\left[\mathrm{L}-(\mathrm{B}-\mathrm{A})^{\prime} \mathrm{H}^{-1} \mathrm{C}\right]^{\prime}\left[\mathrm{N}-\mathrm{BH}^{-1}\left(\mathrm{~B}^{\prime}-\mathrm{A}\right)\right]^{-1}\left[\mathrm{~L}-\mathrm{BH}^{-1} \mathrm{C}\right]
\end{aligned}
$$


Ignorando-se a estimativa ou estimativas das parcelas perdidas, tem-se:

$(4.11 .4 . d) \quad S Q R=\sum_{k j i}^{\prime} y_{k j i}^{2}-S Q P(1)$

onde $\sum_{k j i}^{\prime} y_{k j i}^{2}=$ somatōrio dos quadrados das parcelas existentes.

$$
\text { Somando-se e subtraindo-se uma correção, (Corr (usual)), }
$$

da expressão $(4 \cdot 11.4 . d$ ), e ainda substituindo-se a SQP (1) pela expressão $(4.11 .4 . c)$, tem-se:

$$
\begin{aligned}
(4.11 .4 . e) \quad \mathrm{SQR}= & \left(\sum_{k j i}^{\prime} \mathrm{y}_{k j i}^{2}-\operatorname{Corr}_{(\text {usual })}\right)-\hat{\tau}^{\prime} \mathrm{Q}- \\
& -\left[\mathrm{L}-(\mathrm{B}-\mathrm{A})^{\prime} \mathrm{H}^{-1} \mathrm{C}^{\prime} \cdot\left[\mathrm{N}-\mathrm{BH}^{-1}\left(\mathrm{~B}^{\prime}-\mathrm{A}\right)\right]^{-1}\left[\mathrm{~L}-\mathrm{BH}^{-1} \mathrm{C}\right]-\right. \\
& -\left[\mathrm{C}^{\prime} \mathrm{H}^{-1} \mathrm{C}-\operatorname{Corr} \text { (usual) }\right]
\end{aligned}
$$

onde, $\operatorname{Corr}_{\text {(usual) }}=\frac{G^{2}}{b K-1}$;

$$
G^{*}=\text { total geral das parcelas existentes. }
$$

A expressão (4.11.4.e) é, portanto, a correta soma de quadrados do resíduo, ou seja:

$$
\begin{aligned}
(4.11 .4 . \mathrm{f}) \quad \mathrm{SQR}=\mathrm{SQTotal}_{(\text {usual })} & -\mathrm{SQT}_{(\mathrm{aj})}-\mathrm{SQL}_{(\mathrm{aj})}- \\
& -\mathrm{SQC}_{\text {(usual) }}
\end{aligned}
$$




\subsection{2 - Matriz de Dispersão}

A matriz de dispersão $D$ das estimativas dos parâmetros $\bar{e}$, por definição:

$$
D=E\left\{[\hat{B}-E(\hat{B})][\hat{B}-E(\hat{B})]^{\prime}\right\},
$$

onde $\hat{\beta}$ é dado em (4.4.b), ou seja:

$$
\widehat{B}=F_{1}^{-1} X^{\prime} Y \text {. }
$$

Como no modelo (4.1.b) tem-se que:

$(4.12 . a)$

$$
\left\{\begin{array}{l}
Y=X \beta+\varepsilon \\
\varepsilon \cap N\left(\Phi, \sigma^{2} I_{n}\right)
\end{array}\right.
$$

logo:

$$
E(\hat{B})=E\left[F_{1}^{-1} X^{\prime} X B+F^{-1} X^{\prime} \varepsilon\right]
$$

Pelas propriedades de esperança e por (4.12.a), tem-

-se:

$$
E(\hat{B})=F_{1}^{-1} X^{\prime} X \beta+\Phi,
$$

logo:

$$
D=E\left\{\left[F_{1}^{-1} X^{\prime} \varepsilon\right]\left[F_{1}^{-1} X^{\prime} \varepsilon\right]^{\prime}\right\}
$$

Ainda considerando-se (4.12.a), tem-se:

$(4.12 . b)$

$$
D=F_{1}^{-1} X^{\prime} X\left(F_{1}^{-1}\right)^{\prime} \sigma^{2}
$$


4.13 - Variāncia da Estimativa de Um Contraste entre Duas Médias de Tratamentos Ajustadas para Linhas e Colunas

As estimativas das médias de tratamentos ajustadas pa ra linhas e colunas são obtidas por:

(4.13.a) $\quad \widehat{m}_{i}=\widehat{m}+\hat{t}_{i} ; \quad(i=1,2, \ldots, v)$,

onde: $\widehat{\mathrm{m}}=\frac{G}{\mathrm{bK}}$.

De (4.13.a) obtëm-se:

$(4.13 . b)$

$\hat{m}_{i}-\hat{m}_{i^{\prime}}=\hat{t}_{i}-\hat{t}_{i^{\prime}} \quad$.

Aplicando as propriedades de variância em (4.13.b),

tem-se:

$(4.13 . c)$

$$
V\left(\hat{t}_{i}-\hat{E}_{i^{\prime}}\right)=V\left(\hat{t}_{i}\right)+V\left(\hat{t}_{i}\right)-2 \operatorname{cov}\left(\hat{E}_{i}, \hat{t}_{i}{ }^{\prime}\right) .
$$

De posse da matriz de dispersão (4.12.b), determina-se $(4.13 . c)$.

Seja P' $\bar{B}$ uma função linear dos parâmetros, então a $V\left(P^{i} \hat{\beta}\right) \bar{e}$, por definição:

$$
\begin{aligned}
& V\left(P^{\prime} \hat{B}\right)=E\left\{\left[P^{\prime} \hat{B}-E\left(P^{\prime} \hat{B}\right)\right]\left[P^{\prime} \hat{B}-E\left(P^{\prime} \hat{B}\right)\right]^{\prime}\right\} \\
& V\left(P^{\prime} \hat{B}\right)=E\left\{P^{\prime}[\hat{B}-E(\hat{B})][\hat{B}-E(\hat{B})]^{\prime} P\right\} .
\end{aligned}
$$

Logo:

(4.13.d)

$$
V\left(P^{\prime} \widehat{B}\right)=P^{\prime} D P \quad .
$$


Se $P^{\prime} \hat{B}$ for uma função linear estimāvel, ou seja, exis te $\lambda$ tal que: $P^{\prime}=\lambda^{\prime}\left(X^{\prime} X\right)$, então,

$$
\begin{aligned}
& V\left(P^{\prime} \widehat{B}\right)=\lambda^{\prime}\left(X^{\prime} X\right) F_{1}^{-1}\left(X^{\prime} X\right)\left(F_{1}^{-1}\right)^{\prime}\left(X^{\prime} X\right) \lambda \sigma^{2} . \\
& \text { Mas, }\left(X^{\prime} X\right) F_{1}^{-1}\left(X^{\prime} X\right)=\left(X^{\prime} X\right), \text { logo, } \\
& (4.13 . e) \\
& V\left(P^{\prime} \widehat{B}\right)=P^{\prime} F_{1}^{-1} P \sigma^{2} . \\
& \text { Por (4.13.e) facilmente então se determina a } V\left(\hat{t}_{i}-\widehat{t}_{i^{\prime}}\right) \text {, } \\
& \text { dado que } \hat{\beta}^{\prime}=\left[\hat{\tau}^{\prime}, \bar{l}^{\prime}, \hat{c}^{\prime}\right] \text {. }
\end{aligned}
$$


Tornando minima a SQR deduziram-se fórmulas para as estimativas das parcelas perdidas.

Considerou-se neste trabalho o caso de perda de uma e de duas parcelas na mesma linha.

As correções das somas de quadrados foram obtidas atravēs do método do resỉduo condicional.

Para maior clareza serā deduzido detalhadamente a análise estatística de um delineamento em Quadrado de Youden, para cada caso seguinte:

5.1 - Caso de uma parcela perdida, ignorando-se a mesma.

5.2 - Caso de uma parcela perdida, considerando-se a sua estimativa.

5.3 - Caso de duas parcelas perdidas na mesma linha, sendo que o tratamento correspondente a uma das parcelas perdidas não 
39.

figure dentre as parcelas remanescentes da outra coluna em que ocorreu a perda e vice-versa. As parcelas perdidas são ignoradas .

5.4 - Caso de duas parcelas perdidas na mesma linha, sendo que o tratamento correspondente a uma das parcelas perdidas não figura dentre as parcelas remanescentes da outra coluna em que ocorreu a perda e vice-versa. As estimativas das parce las perdidas são consideradas.

5.5 - Caso de duas parcelas perdidas na mesma linha, sendo que apenas em uma das colunas com parcela perdida figure o tra tamento correspondente à parcela perdida da outra coluna. As parcelas perdidas são ignoradas.

5.6 - raso de duas parcelas perdidas na mesma linha, sendo que penas em uma das colunas com parcela perdida figura o tra tamento correspondente à parcela perdida da outra coluna. As estimativas das parcelas perdidas são consideradas.

5.7 - Caso de duas parcelas perdidas na mesma linha, sendo que em cada uma das colunas com parcela perdida figure o trata mento correspondente à parcela perdida da outra coluna e vice-versa, As parcelas perdidas são ignoradas.

5.8 - Caso de duas parcelas perdidas na mesma linha, sendo que em cada uma das colunas com parcela perdida figure o trata mento correspondente à parcela perdida da outra coluna e vice-versa. As estimativas das parcelas perdidas são cons $\underline{i}$ deradas . 
5.1 - Caso de Uma Parcela Perdida, Ignorando-se a Mesma

Seja um experimento em Quadrado de Youden, onde A, B, $C$ e D são os tratamentos

$(5.1 . a)$

\begin{tabular}{|c|c|c|c|c|c|}
\hline \multirow{2}{*}{ LINHAS } & \multicolumn{4}{|c|}{ COLUNAS } & \multirow{2}{*}{$\begin{array}{l}\text { TOTAIS DE } \\
\text { L INHAS }\end{array}$} \\
\hline & (1) & (2) & (3) & (4) & \\
\hline I & $x(A)$ & $B$ & C & $\mathrm{D}$ & $L^{*}$ \\
\hline II & B & $C$ & D & A & \\
\hline III & C & $\mathrm{D}$ & A & B & \\
\hline $\begin{array}{l}\text { TOTAIS DE } \\
\text { COLUNAS }\end{array}$ & $C^{*}$ & & & & $G^{*}$ \\
\hline
\end{tabular}

onde:

$\mathrm{x}$ : representa a parcela perdida que continha o tratamento A;

$L^{*}$ : total das parcelas existentes ra linha (I);

$C^{*}$ : total das parcelas existentes na coluna (1);

G*: total geral das parcelas disponíveis;

$\mathrm{T}^{*}$ : total das parcelas existentes com o tratamento A.

5.1.1 - Sistema de equações normais e estimativas dos parā metros

O sistema de equações normais é o descrito em (4.3.a),

onde: 
$R_{v v}=\left[\begin{array}{cccc}2 & 0 & 0 & 0 \\ 0 & 3 & 0 & 0 \\ 0 & 0 & 3 & 0 \\ 0 & 0 & 0 & 3\end{array}\right] ; \quad E_{b i}=\left[\begin{array}{ccc}0 & 1 & 1 \\ 1 & 1 & 1 \\ 1 & 1 & 1 \\ 1 & 1 & 1\end{array}\right] ; \quad W_{v b}=\left[\begin{array}{cccc}0 & 0 & 1 & 1 \\ 1 & 1 & 0 & 1 \\ 1 & 1 & 1 & 0 \\ 0 & 1 & 1 & 1\end{array}\right]$

$\mathrm{N}_{\mathrm{KK}}=\left[\begin{array}{ccc}3 & 0 & 0 \\ 0 & 4 & 0 \\ 0 & 0 & 4\end{array}\right] \quad \mathrm{B}_{\mathrm{bK}}^{\prime}=\left[\begin{array}{ccc}0 & 1 & 1 \\ 1 & 1 & 1 \\ 1 & 1 & 1 \\ 1 & 1 & 1\end{array}\right] ; \quad \mathrm{H}_{\mathrm{bb}}=\left[\begin{array}{cccc}2 & 0 & 0 & 0 \\ 0 & 3 & 0 & 0 \\ 0 & 0 & 3 & 0 \\ 0 & 0 & 0 & 3\end{array}\right]$

$\hat{\tau}^{\prime}=\left[\hat{t}^{*}, \hat{t}_{B}, \hat{t}_{C}, \hat{t}_{D}\right]$

$\bar{l}^{\prime}=\left[\bar{l}^{*}, \bar{l}_{2}, \bar{l}_{3}\right]$

$\hat{c}^{\prime}=\left[\hat{c}^{*}, \hat{c}_{2}, \hat{c}_{3}, \hat{c}_{4}\right]$

$\mathrm{T}^{\prime}=\left[\mathrm{T} *, \mathrm{~T}_{\mathrm{B}}, \mathrm{T}_{\mathrm{C}}, \mathrm{T}_{\mathrm{D}}\right]$

$\mathrm{L}^{\prime}=\left[\mathrm{L} *, \mathrm{~L}_{2}, \mathrm{~L}_{3}\right]$

$C^{\prime}=\left[C^{*}, C_{2}, C_{3}, C_{4}\right]$

Nota-se que para o particular exemplo literal (5.1.a),

tem-se:

$$
x_{1}^{\prime} x_{1}=x_{3}^{\prime} x_{3} \quad \text { e } \quad x_{1}^{\prime} x_{2}=x_{3}^{\prime} x_{2}
$$

ou seja:

$$
\mathrm{R}_{v v}=\mathrm{H}_{b b} \quad \text { e } \quad E_{b k}=B_{b K}^{\prime} \text {. }
$$


Considerando-se as restrições $\sum_{j} \hat{c}_{j}=0$ e $\sum_{k} \bar{l}_{k}=0$, as estimativas dos parâmetros são dadas por $\hat{B}^{j}=\left(F_{1}\right)^{-1} X^{\prime} Y,(4.4 . b)$, ou seja:

$(5.1 .1 . a) \quad \widehat{B}=\left[\begin{array}{ccccccccccc}2 & 0 & 0 & 0 & -1 & 0 & 0 & -1 & -1 & 0 & 0 \\ 0 & 3 & 0 & 0 & 0 & 0 & 0 & 0 & 0 & -1 & 0 \\ 0 & 0 & 3 & 0 & 0 & 0 & 0 & 0 & 0 & 0 & -1 \\ 0 & 0 & 0 & 3 & 0 & 0 & 0 & -1 & 0 & 0 & 0 \\ 0 & 1 & 1 & 1 & 3 & 0 & 0 & -1 & 0 & 0 & 0 \\ 1 & 1 & 1 & 1 & 0 & 4 & 0 & 0 & 0 & 0 & 0 \\ 1 & 1 & 1 & 1 & 0 & 0 & 4 & 0 & 0 & 0 & 0 \\ 0 & 1 & 1 & 0 & -1 & 0 & 0 & 2 & 0 & 0 & 0 \\ 0 & 1 & 1 & 1 & 0 & 0 & 0 & 0 & 3 & 0 & 0 \\ 1 & 0 & 1 & 1 & 0 & 0 & 0 & 0 & 0 & 3 & 0 \\ 1 & 1 & 0 & 0 & 0 & 0 & 0 & 0 & 0 & 0 & 3\end{array}\right]_{T_{C}}^{-1}\left[\begin{array}{c}T^{*} \\ T_{B} \\ T^{*} \\ L_{2} \\ L_{3} \\ C_{*} \\ C_{2} \\ C_{3} \\ C_{4}\end{array}\right]$

5.1 .2 - Cālculo das $S_{(1)}, S_{(2)}$ e $S_{(3)}$

$\operatorname{A~SQP}_{(1)},(4.5 . a), \bar{e}$ obtida com a solução (5.1.1.a).

Considerando-se o sistema de equações normais (4.5.e), cuja solução, $(4.5 \cdot g), \bar{e}:$ 
43.

$(5.1 .2 . a) \quad \tilde{B}^{*}=\left[\begin{array}{ccccccc}3 & 0 & 0 & -1 & 0 & 0 & 0 \\ 0 & 4 & 0 & 0 & 0 & 0 & 0 \\ 0 & 0 & 4 & 0 & 0 & 0 & 0 \\ 0 & 1 & 1 & 2 & 0 & 0 & 0 \\ 1 & 1 & 1 & 0 & 3 & 0 & 0 \\ 1 & 1 & 1 & 0 & 0 & 3 & 0 \\ 1 & 1 & 1 & 0 & 0 & 0 & 3\end{array}\right]^{-1}\left[\begin{array}{l}L^{*} \\ L \\ L \\ C \\ C \\ C \\ C\end{array}\right]$

determina-se a $\mathrm{SQP}_{(2)^{\circ}}$.

Determina-se a $\operatorname{SQP}_{(3)},(4.5 . n)$, com a solução:

$(5.1 .2 . b)$

$$
\left[\begin{array}{c}
\hat{c}^{*} \\
\hat{c} \\
\hat{c} \\
\hat{c}
\end{array}\right]=\left[\begin{array}{llll}
2 & 0 & 0 & 0 \\
0 & 3 & 0 & 0 \\
0 & 0 & 3 & 0 \\
0 & 0 & 0 & 3
\end{array}\right]^{-1}\left[\begin{array}{l}
c * \\
c \\
c \\
c
\end{array}\right]
$$

5.1.3 - Anārise da variāncia

o quadro da análise da variância é dado a seguir:

\begin{tabular}{lclll}
\hline C.V. & G.L. & S.Q. & Q.M. & F \\
\hline Tratamentos $(a j)$ & $\mathrm{v}-1=3$ & $\mathrm{SQ}(\mathrm{T} \mid \ell, \mathrm{C})$ & $\mathrm{QM}(\mathrm{T} \mid \ell, \mathrm{c}) \quad(\mathrm{a}) \mathrm{a} / \mathrm{b}$ \\
Linhas $(\mathrm{aj})$ & $\mathrm{K}-1=2$ & $\mathrm{SQ}(\mathrm{L} \mid \mathrm{c})$ & $\mathrm{QM}(\mathrm{L} \mid \mathrm{c})$ & \\
Colunas & $\mathrm{b}-1=3$ & $\mathrm{SQC}$ (usual) & $\mathrm{QMC}$ (usual) \\
Resíduo & $[(\mathrm{b}-1)(\mathrm{K}-2)-1)]=2$ & $\mathrm{SQR}$ & $\mathrm{QMR}$ & $(\mathrm{b})$
\end{tabular}

Total

$[(\mathrm{bK}-1)-1]=10$ 
onde:

$$
\begin{aligned}
& \mathrm{SQ}(\mathrm{T} \mid \ell, C)=\mathrm{SQP}_{(1)}-\mathrm{SQP}_{(2)} \text { ou } \mathrm{SQ}(\mathrm{T} \mid \ell, \mathrm{C})=\hat{\tau}^{\prime} \mathrm{Q},(4.11 .1 . \mathrm{b}) ; \\
& \mathrm{SQ}(\mathrm{L} \mid \mathrm{C})=\mathrm{SQP}_{(2)}-\mathrm{SQP}_{(3)} \text {, ou obtida atravës da expressão } \\
& (4.11 .2 . h) \text {; } \\
& S Q C_{(\text {usual) }}=\sum_{j} \frac{C_{j}^{2}}{h_{j}}-\operatorname{Corr}_{(\text {usual) }} ; \operatorname{Corr}_{\text {(usual) }}=\frac{G^{2}}{b k-1} ; \\
& \text { SQTotal (usual) }=\underset{k j i}{\sum^{\prime}} y_{k j i}^{2}-\operatorname{Corr}_{\text {(usual) }} \text {; }
\end{aligned}
$$

e a SQR é obtida por diferença entre a SQTotal (usual) e as demais so mas de quadrados.

5.1.4 - Variāncia da estimativa de um contraste entre duas médias de tratamentos, ajustadas para linhas e colunas

$$
\begin{aligned}
& \text { Como visto em }(4.13 . e) \text {, tem-se: } \\
& \qquad v\left(\hat{E}_{i}-\hat{E}_{i}\right)=\mathrm{P}^{\prime} \mathrm{F}_{1}^{-1} \mathrm{P} \sigma^{2},
\end{aligned}
$$

onde $F_{!}^{-i}$ è dado em $(5.1 .1 . a)$ e QMR $=\sigma^{2}$. 
5.2 - Caso de Uma Parcela Perdida, Considerando-se a Sua Estimativa

5.2.1 - Fórmula para a estimativa de uma parcela perdida

$$
\text { Considerando-se o experimento (5.1.a) e as somas de }
$$

quadrados dadas em 4.9, tem-se:

$\mathrm{SQT}_{(\mathrm{x})}=\frac{3}{8}\left\{\begin{array}{l}{\left[\mathrm{T}^{*}+\mathrm{x}-\frac{1}{\mathrm{~K}}\left(\mathrm{C}^{*}+\mathrm{x}+\mathrm{C}_{3}+\mathrm{C}_{4}\right)\right]^{2}+} \\ +\left[\mathrm{T}_{\mathrm{B}}-\frac{1}{\mathrm{~K}}\left(\mathrm{C}^{*}+\mathrm{x}+\mathrm{C}_{2}+\mathrm{C}_{4}\right)\right]^{2}+ \\ +\left[\mathrm{T}_{\mathrm{C}}-\frac{1}{\mathrm{~K}}\left(\mathrm{C}^{*}+\mathrm{x}+\mathrm{C}_{2}+\mathrm{C}_{3}\right)\right]^{2}+ \\ +\left[\mathrm{T}_{\mathrm{D}}-\frac{1}{\mathrm{~K}}\left(\mathrm{C}_{2}+\mathrm{C}_{3}+\mathrm{C}_{4}\right)\right]^{2}\end{array} \mid\right.$

$\operatorname{SQCol}_{(x)}=\frac{\left(C^{*}+x\right)^{2}}{K}+\frac{C_{2}^{2}}{K}+\frac{C_{3}^{2}}{K}+\frac{C_{4}^{2}}{K}-\operatorname{Corr}(x) ;$

$\operatorname{SQL}_{(x)}=\frac{\left(L^{*}+x\right)}{b}+\frac{L_{2}^{2}}{b}+\frac{L_{3}^{2}}{b}-\operatorname{Corr}(x) \quad ;$

$\operatorname{SQTotal}_{(\mathrm{x})}=\mathrm{x}+\sum_{k j i}^{1} \mathrm{y}_{k j i}^{2}-\operatorname{Corr}(\mathrm{x}) \quad ;$

$S Q R_{(x)}=\operatorname{SQTotal}(x)-S Q L_{(x)}-S Q C_{(x)}-S Q T(x)$. 
Tornando mínima a $S Q R(x)$, obtēm-se a fórmula para a estimativa de uma parcela perdida, que para um caso geral, é dada por:

$(5.2 .1 . a)$

$$
x=\frac{\lambda K^{*}+(\lambda v+1-K) C^{*}-K\left[\sum_{j}^{\prime} C_{j}+\sum_{i}^{\prime} T_{i}-(K-1) T^{*}\right]}{K(K-1)(K-2)}
$$

onde:

$$
\begin{aligned}
& L^{*}, C^{*} \text { e } T^{*}: \text { dados em } 5.1 ; \\
& \begin{aligned}
\lambda, & K, \text { v: dados em } 4 ; \\
\sum^{\prime} & C_{j}: \text { somatório dos totais de colunas onde aparece o } \\
& \text { tratamento correspondente à parcela perdida; } \\
\sum^{\prime} T_{i}: & \text { somatório dos totais de todos os tratamentos que } \\
& \text { aparecem na coluna que contém a parcela perdida. }
\end{aligned}
\end{aligned}
$$

Este resultado se assemelha ao apresentado por $\mathrm{COCH}-$ RAN e COX (1957).

\subsection{2 - Förmulas para as correções das somas de quadrados de tratamentos e de linhas: $U_{T}$ e $U_{L}$}

Considerando-se (5.1.a) um delineamento em blocos ao acaso, obtëm-se:

$$
\begin{aligned}
& \operatorname{SQC}_{(y)}=\frac{\left(C^{*}+y\right)^{2}}{K}+\frac{C_{2}^{2}}{K}+\frac{C_{3}^{2}}{K}+\frac{C_{4}^{2}}{K}-\operatorname{Corr}(y) \\
& \operatorname{SQL}_{(y)}=\frac{\left(L^{*}+y\right)^{2}}{b}+\frac{L_{2}^{2}}{b}+\frac{L_{3}^{2}}{b}-\operatorname{Corr}(y)
\end{aligned}
$$




$$
\begin{aligned}
& \operatorname{SQTotal}_{(y)}=y^{2}+\underset{k j i}{\sum_{k j i}^{\prime}} y_{k j}^{2}-\operatorname{Corr}(y), \text { onde } \operatorname{Corr}(y)=\frac{(G *+y)}{b K} \\
& \operatorname{SQR}_{(y)}=\operatorname{SQTotal}_{(y)}-\operatorname{SQC}_{(y)}-\operatorname{SQL}_{(y)} \cdot
\end{aligned}
$$

Tornando mínima a $\mathrm{SQR}_{(\mathrm{y})}$, obtém-se a fórmula para a estimativa da parcela perdida de um delineamento em blocos ao acaso, que para um caso geral è dada por:

$$
\text { (5.2.2.a) } \quad y=\frac{k L *+b C *-G *}{(k-1)(b-1)} \text {. }
$$

Sabe-se que:

$(5.2 .2 . b)$

$$
\mathrm{SQR}_{(\mathrm{y})}-\mathrm{SQR}_{(\mathrm{x})}=\mathrm{SQP}_{(1)}-\mathrm{SQP}_{(2)}=\mathrm{SQT}_{(\mathrm{aj})} .
$$

Desenvolvendo-se a expressão (5.2.2.b), obtëm-se:

$(5.2 .2 . c)$

$$
\mathrm{SQT}_{(\mathrm{aj})}=\mathrm{SQT}_{(\mathrm{x})}-\mathrm{U}_{\mathrm{T}},
$$

onde $\mathrm{U}_{\mathrm{T}} \overrightarrow{\mathrm{e}}$ dado por:

$$
(5.2 .2 . e) \quad U_{T}=\frac{(b-1)(K-1)}{6 K}(x-y)^{2},
$$

onde $x$ e y são dados em (5.2.1.a) e (5.2.2.a), respectivamente.

Analisando-se a fórmula (5.2.2.e) verifica-se que es ta serā sempre positiva, o que indica que a $\mathrm{SQT}_{(\mathrm{x})}$ estará sempre su perestimada. Considerando-se (5.1.a) um delineamento inteiramente ca sualizado, tem-se: 


$$
\begin{aligned}
\operatorname{SQC}_{(z)} & =\frac{\left(C^{*}+z\right)^{2}}{K}+\frac{C_{2}^{2}}{K}+\frac{C_{3}^{2}}{K}+\frac{C_{4}^{2}}{K}-\operatorname{Corr}(z) \\
\operatorname{SQTotal}_{(z)} & =z^{2}+\sum_{k j i} y_{k j i}^{2}-\operatorname{Corr}(z) \\
\operatorname{SQR}_{(z)} & =\operatorname{SQTotal}_{(z)}-\operatorname{SQC}_{(z)} .
\end{aligned}
$$

Tornando mínima a $\mathrm{SQR}_{(z)}$, obtém-se a Éórmula para a estimativa da parcela perdida de um delineamento inteiramente casua lizado, que para um caso geral é dada por:

$$
(5.2 .2 . E) \quad z=\frac{C^{*}}{K-1} .
$$

Sabe-se que:

(5.2.2.g) $\quad \mathrm{SQR}_{(z)}-\mathrm{SQR}_{(\mathrm{y})}=\mathrm{SQP}_{(2)}-\mathrm{SQP}_{(3)}=\mathrm{SQL}_{(\mathrm{aj})} \cdot$

$$
\text { Desenvolvendo-se a expressão }(5.2 .2 . g) \text { e somando-se }
$$

e subtraindo-se da mesma $\frac{\left(L^{*}+x\right)^{2}}{b} e \frac{\left(G^{*}+x\right)^{2}}{b K}$, obtēm-se:

$$
S_{(a j)}=S Q L(x)-U_{L} \text {, }
$$

onde:

(5.2.2.i) $U_{L}=\frac{K-1}{b k}\left\{\left[x^{2}-(b-1) y^{2}+b z^{2}\right]+2 x \frac{\left(K L *-G^{*}\right)}{K-1}\right\}$. 


\subsection{3 - Anālise da variância}

O quadro da anālise da variância para um caso geral

é o dado a seguir:

\begin{tabular}{|c|c|c|c|c|}
\hline$C . V$. & G.L. & $S \cdot Q$. & Q.M. & $\mathrm{E}$ \\
\hline Tratamentos $(a j)$ & $v-1$ & 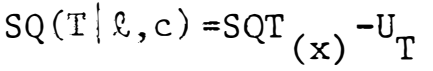 & $\mathrm{QM}(\mathrm{T} \mid \ell, \mathrm{c}) \quad(\mathrm{a})$ & $a / b$ \\
\hline Linhas $(a j)$ & $\mathrm{K}-1$ & $S Q(L \mid C)=S Q L(x)^{-U_{L}}$ & $Q M(L \mid C)$ & \\
\hline Colunas & $b-1$ & $\mathrm{SQC}_{\text {(usual) }}$ & QMC (usual) & \\
\hline Resíduo & $v-1)(K-2)-1$ & $\mathrm{SQR}$ & $Q M R$ & \\
\hline Total & $(b K-1)-1$ & SQTotal (usual) & & \\
\hline
\end{tabular}

onde a $\mathrm{SQR}$ é obtida por diferença entre a SQTotal (usual) e as demais somas de quadrados.

5.2.4 - Comparação entre duas mēdias de tratamentos, ajustadas para linhas e colunas

Como visto em $(5.1 .4)$, tem-se

$$
V\left(\hat{E}_{i}-\hat{E}_{i}{ }^{\prime}\right)=P^{\prime} F_{1}^{-1} P \sigma^{2}
$$

Serā definido, a seguir, algumas expressões que serão utilizadas para os casos de duas parcelas perdidas na mesma linha. 
$\sum_{k j i}^{\prime} y_{k j i}^{2}=$ somatório dos quadrados das parcelas existentes;

$G^{*}=$ total geral das parcelas disponiveis;

$L^{*}=$ total da 1 inha que contēm as parcelas perdidas;

$x_{1}, x_{2}=$ representam as parcelas perdidas no delineamento em Quadrado de Youden;

$y_{1}, y_{2}=$ representam as parcelas perdidas admitindo-se o deli neamento em blocos casualizados;

$z_{1}, z_{2}=$ representam as parcelas perdidas admitindo-se o deli neamento inteiramente casualizado;

$T_{1}^{*}=$ total do tratamento correspondente à parcela perdida $\mathrm{x}_{1}$;

$C_{1}^{*}=$ total da coluna que contēm a parcela perdida $x_{1}$;

$T_{2}^{*}=$ total do tratamento correspondente à parcela perdida $\mathrm{x}_{2}$;

$C_{2}^{*}=$ total da coluna que contém a parcela perdida $x_{2}$;

$\mathrm{T}_{1}^{\prime}=$ somatório do total de todos os tratamentos que apare cem na coluna que contēm a parcela perdida $x_{1}$;

$\mathrm{T}_{2}^{\prime}=$ somatōrio do total de todos os tratamentos que apare cem na coluna que contēm a parcela perdida $x_{2}$;

$Q_{1}=$ somatório do total de todas as colunas que apresentam o tratamento correspondende ao da parcela perdida $x_{1}$; $Q_{2}=$ somatório do total de todas as colunas que apresentam o tratamento correspondente ao da parcela perdida $x_{2}$;

$Q_{1}^{\prime}=$ total dos valores $Q_{1}$ para os outros tratamentos que aparecem na coluna que contēm $x_{1}$; 


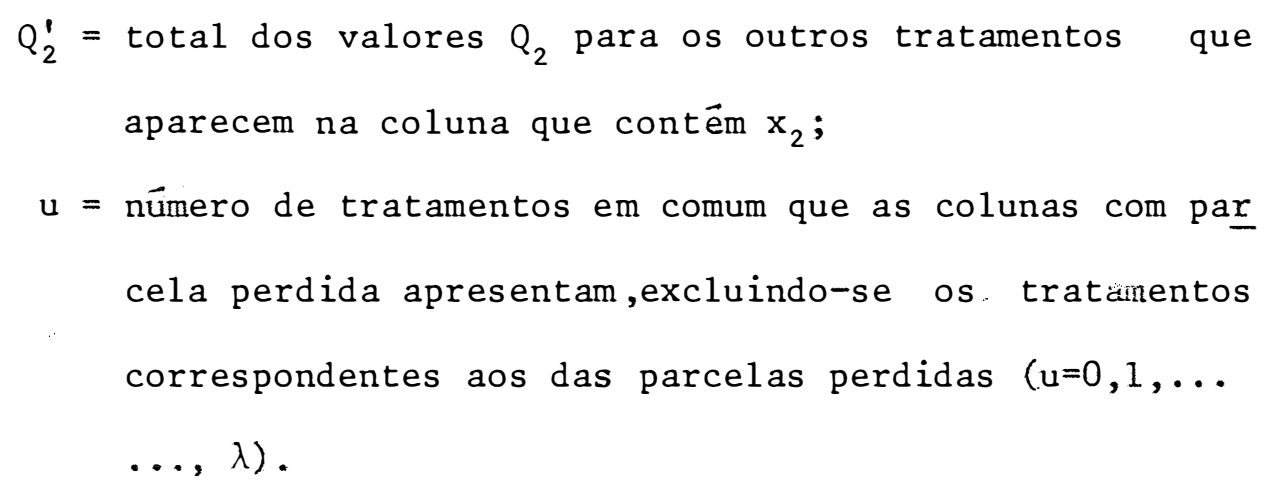

Deve-se salientar ainda que $Q_{1}$ é um somatório de $K$ colunas, portanto inclui sempre $C_{1}^{*}$. $O$ mesmo ocorre com $Q_{2}$, que sempre inclui $\mathrm{C}_{2}^{*}$.

A $S Q R$ de todos os quadros das anālises de variância são obtidas por diferença entre a SQTotal (usual) e as demais corretas somas de quadrados.

5.3 - Caso de Duas Parcelas Perdidas na Mesma Linha, sendo que o Tratamento Correspondente à Parcela Perdida de uma Coluna não Figure Dentre as Parcelas Remanescentes da outra Coluna em que Ocorreu a Perda e Vice-Versa. As Parcelas Perdidas são Ignoradas

Seja um experimento em Quadrado de Youden, onde A, B, $C, D, E, F$ e $G$ são os tratamentos: 


\begin{tabular}{|c|c|c|c|c|c|c|c|c|}
\hline LINHAS & (1) & (2) & $\begin{array}{l}C 0 \\
(3)\end{array}$ & $\begin{array}{l}\mathrm{L} \underset{(4)}{\mathrm{U}} \\
\end{array}$ & $\begin{array}{l}S \\
(5)\end{array}$ & (6) & (7) & $\begin{array}{c}\text { TOTAIS } \\
\text { DE LINHAS }\end{array}$ \\
\hline I & $x_{1}(G)$ & A & $x_{2}(B)$ & C & D & $\mathrm{E}$ & F & $L^{*}$ \\
\hline II & A & B & C & D & $\mathrm{E}$ & F & G & \\
\hline III. & C & D & $\mathrm{E}$ & F & G & $\mathrm{A}$ & $\mathrm{B}$ & \\
\hline $\begin{array}{l}\text { TOTAIS } \\
\text { DE } \\
\text { COLUNAS }\end{array}$ & $C_{1}^{*}$ & & $C_{2}^{*}$ & & & & & $G^{*}$ \\
\hline
\end{tabular}

(5.3.a)

Tem-se: $\mathrm{v}=7 ; \mathrm{K}=3 ; \mathrm{b}=7 ; \mathrm{r}=3 ; \lambda=1$ e $\mathrm{u}=1$

5.3.1 - Sistema de equações normais e estimativas dos parā metros

0 sistema de equações normais é o (4.3.a), onde:

$R_{v V}=\left[\begin{array}{ccccccc}3 & 0 & 0 & 0 & 0 & 0 & 0 \\ 0 & 2 & 0 & 0 & 0 & 0 & 0 \\ 0 & 0 & 3 & 0 & 0 & 0 & 0 \\ 0 & 0 & 0 & 3 & 0 & 0 & 0 \\ 0 & 0 & 0 & 0 & 3 & 0 & 0 \\ 0 & 0 & 0 & 0 & 0 & 3 & 0 \\ 0 & 0 & 0 & 0 & 0 & 0 & 2\end{array}\right] ; E_{b K}=\left[\begin{array}{ccc}1 & 1 & 1 \\ 0 & 1 & 1 \\ 1 & 1 & 1 \\ 1 & 1 & 1 \\ 1 & 1 & 1 \\ 1 & 1 & 1 \\ 0 & 1 & 1\end{array}\right]$ 


$$
\mathrm{W}_{\mathrm{vb}}=\left[\begin{array}{ccccccc}
1 & 1 & 0 & 0 & 0 & 1 & 0 \\
0 & 1 & 0 & 0 & 0 & 0 & 1 \\
1 & 0 & 1 & 1 & 0 & 0 & 0 \\
0 & 1 & 0 & 1 & 1 & 0 & 0 \\
0 & 0 & 1 & 0 & 1 & 1 & 0 \\
0 & 0 & 0 & 1 & 0 & 1 & 1 \\
0 & 0 & 0 & 0 & 1 & 0 & 1
\end{array}\right] ; \mathrm{N}_{\mathrm{KK}}=\left[\begin{array}{ccc}
5 & 0 & 0 \\
0 & 7 & 0 \\
0 & 0 & 7
\end{array}\right] ;
$$$$
\mathrm{H}_{\mathrm{bb}}=\left[\begin{array}{cccccc}
2 & 0 & 0 & 0 & 0 & 0 \\
0 & 3 & 0 & 0 & 0 & 0 \\
0 & 0 & 2 & 0 & 0 & 0 \\
0 & 0 & 0 & 3 & 0 & 0 \\
0 & 0 & 0 & 0 & 3 & 0 \\
0 & 0 & 0 & 0 & 0 & 3
\end{array}\right] ; B_{\mathrm{Kb}}=\left[\begin{array}{lllllll}
0 & 1 & 0 & 1 & 1 & 1 & 1 \\
1 & 1 & 1 & 1 & 1 & 1 & 1 \\
1 & 1 & 1 & 1 & 1 & 1 & 1
\end{array}\right]
$$

Considerando-se as restrições $\sum \hat{c}_{j}=0$ e $\sum \widehat{l}_{k}=0$, tem-se as estimativas dos parâmetros, (4.4.b), ou seja:

$$
(5.3 .1 . \mathrm{a}) \quad \hat{B}=\left[\begin{array}{ccc}
{\left[\mathrm{R}_{\mathrm{VV}}\right]} & {\left[\mathrm{E}_{\mathrm{bK}}-\mathrm{A}_{\mathrm{bK}}\right]} & {\left[\mathrm{W}_{\mathrm{vb}}-\mathrm{A}_{\mathrm{vb}}\right]} \\
{\left[\mathrm{E}_{\mathrm{Kb}}^{\prime}\right]} & {\left[\mathrm{N}_{\mathrm{KK}}\right]} & {\left[\mathrm{B}_{\mathrm{Kb}}-\mathrm{A}_{\mathrm{Kb}}\right]} \\
{\left[\mathrm{W}_{\mathrm{bV}}^{\prime}\right]} & {\left[\mathrm{B}_{\mathrm{bK}}^{\prime}-\mathrm{A}_{\mathrm{bK}}\right]} & {\left[\mathrm{H}_{\mathrm{bb}}\right]}
\end{array}\right]^{-1}\left[\begin{array}{c}
\mathrm{T} \\
\mathrm{L}
\end{array}\right]
$$


5.3 .2 - Cālculo das $S_{(1)}, S_{(2)}$ e ${ }^{S Q P}(3)$

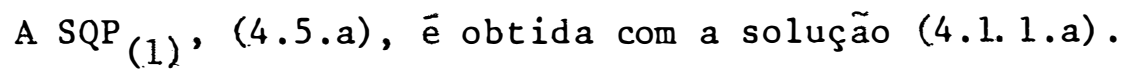
Para determinar-se a $S_{(2)}$ utiliza-se a solução (4.5.g) do sistema (4.5.e), que para o caso è:

$\tilde{\beta}^{*}=\left[\begin{array}{cccccccccc}5 & 0 & 0 & -1 & 0 & -1 & 0 & 0 & 0 & 0 \\ 0 & 7 & 0 & 0 & 0 & 0 & 0 & 0 & 0 & 0 \\ 0 & 0 & 7 & 0 & 0 & 0 & 0 & 0 & 0 & 0 \\ 0 & 1 & 1 & 2 & 0 & 0 & 0 & 0 & 0 & 0 \\ 1 & 1 & 1 & 0 & 3 & 0 & 0 & 0 & 0 & 0 \\ 0 & 1 & 1 & 0 & 0 & 2 & 0 & 0 & 0 & 0 \\ 1 & 1 & 1 & 0 & 0 & 0 & 3 & 0 & 0 & 0 \\ 1 & 1 & 1 & 0 & 0 & 0 & 0 & 3 & 0 & 0 \\ 1 & 1 & 1 & 0 & 0 & 0 & 0 & 0 & 3 & 0 \\ 1 & 1 & 1 & 0 & 0 & 0 & 0 & 0 & 0 & 3\end{array}\right]^{-1}\left[\begin{array}{c}L^{*} \\ L_{2} \\ L_{3} \\ C_{1}^{*} \\ C_{3} \\ C_{2}^{*} \\ C_{4} \\ C_{5} \\ C_{6} \\ C_{7}\end{array}\right]$

Determina-se a $\mathrm{SQP}_{(3)},(4.5 . \mathrm{n})$, com a solução abaixo:

$$
\underline{\tilde{c}}=\mathrm{H}^{-1} \mathrm{C}
$$

5.3.3 - Anālise da variāncia

o quadro da anālise da variância é o dado a seguir. 


\begin{tabular}{|c|c|c|c|c|}
\hline C.V. & G.L. & $S . Q$. & Q.M. & $\mathrm{F}$ \\
\hline Tratamentos $(a j)$ & $v-1=6$ & $\mathrm{SQ}(\mathrm{T} \mid \ell, c)=\mathrm{SQP}_{(1)^{-S Q P}}(2)$ & $\mathrm{QM}(\mathrm{T} \mid \ell, \mathrm{c})$ & (a) $a / b$ \\
\hline Linhas $(a j)$ & $K-1=2$ & $\mathrm{SQ}(\mathrm{L} \mid \mathrm{c})=\mathrm{SQP}_{(2)}{ }^{-\mathrm{SQP}}(3)$ & $\mathrm{QM}(L \mid c)$ & \\
\hline Colunas & $b-1=6$ & $\mathrm{SQC}_{\text {(usual) }}$ & QMC (usual) & \\
\hline Resíduo & $(K-2)-2]=4$ & $S Q R$ & QMR (b) & \\
\hline$[(b K$ & {$[(b K-1)-2]=18$} & SQTotal (usual) & & \\
\hline
\end{tabular}

A $S Q(T \mid \ell, c)$ também pode ser obtida por $(4.11 .1 . b)$ e a $\mathrm{SQ}(\mathrm{L} \mid \mathrm{C})$ por $(4.11 .2 . \mathrm{e})$, respectivamente.

5.3.4 - Variãncia da estimativa de um contraste entre médias de tratamentos, ajustadas para linhas e colunas

Como visto em (4.13.e), tem-se:

$$
V\left(\hat{t}_{i}-\hat{t}_{i}\right)=P^{\prime} F_{1}^{-1} P \sigma^{2} \text {, }
$$

onde $\mathrm{F}^{-1}$ é dado em (5.3.1.a) e QMR $\risingdotseq \sigma^{2}$. 
5.4 - Caso de Duas Parcelas Perdidas na Mesma Linha, sendo que o Tratamento Correspondente à Parcela Perdida de Uma Coluna Não Figure Dentre as Parcelas Remanescentes da Outra Coluna em que Ocorreu a Perda e Vice-Versa. As Estimativas das Parce las Perdidas São Consideradas

5.4.1 - Fórmulas para as estimativas das parcelas perdidas

Como descrito em 4.9, tem-se:

$$
\begin{aligned}
\operatorname{SQR}_{\left(x_{1}, x_{2}\right)=\operatorname{SQTotal}_{\left(x_{1}, x_{2}\right)}}-\operatorname{SQL}_{\left(x_{1}, x_{2}\right)-\operatorname{SQC}_{\left(x_{1}, x_{2}\right)}-} \\
-\operatorname{SQT}_{\left(x_{1}, x_{2}\right)}
\end{aligned}
$$

Minimizando-se a $\mathrm{SQR}_{\left(\mathrm{x}_{1}, \mathrm{x}_{2}\right)}$, ou seja, diferenciando a

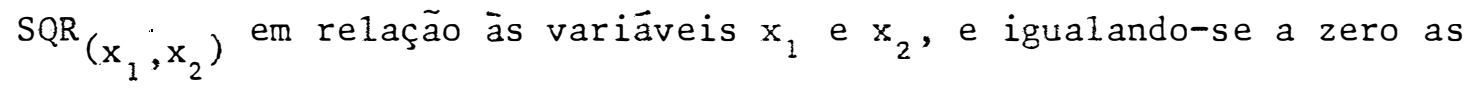
diferenciais parciais, obtēm-se um sistema de equações determinado.

A solução desse sistema são as estimativas das parceLas perdidas:

$$
\begin{aligned}
& K(K-1)(K-2) x_{1}-[\lambda(K-1)+u] x_{2}=\left[\begin{array}{c}
\lambda K L * \lambda b C_{1}^{*}+K(K-1) T_{1}^{*}- \\
-\lambda G^{*}-K T_{1}^{\prime}- \\
-(K-1) Q_{1}+Q_{1}^{\prime}
\end{array}\right] \\
& K(K-1)(K-2) x_{2}-[\lambda(K-1)+u] x_{1}=\left[\begin{array}{c}
\lambda K L *+\lambda b C_{2}^{*}+K(K-1) T_{2}^{*}- \\
-\lambda G^{*}-K T_{2}^{\prime}- \\
-(K-1) Q_{2}+Q_{2}^{\prime}
\end{array}\right]
\end{aligned}
$$

$(5.4 .1 . a)$ 
5.4.2 - Förmulas para as correções das somas de quadrados de tratamentos e de Iinhas: $U_{T}$ e $U_{L}$

Como jà descrito em 4.10, tem-se:

$$
\begin{aligned}
& \operatorname{SQR}_{\left(y_{1}, y_{2}\right)}=\operatorname{SQTotal}_{\left(y_{1}, y_{2}\right)}-\operatorname{SQC}_{\left(y_{1}, y_{2}\right)}-\operatorname{SQL}_{\left(y_{1}, y_{2}\right)} . \\
& \text { Minimizando-se a } S Q R\left(y_{1}, y_{2}\right) \text {, ou seja, igualando-se a }
\end{aligned}
$$
zero as suas diferenciais parciais, obtém-se um sistema de equações. A solução deste são as estimativas das parcelas perdidas de um deli neamento em blocos ao acaso.

$(5.4 .2 . a)$

$$
\left\{\begin{array}{l}
(b-1) y_{1}-y_{2}=\frac{k L^{*}-b C_{1}^{*}-G^{*}}{k-1} \\
(b-1) y_{2}-y_{1}=\frac{k L^{*}-b C_{2}^{*}-G^{*}}{k-1}
\end{array}\right.
$$

Como dado em (4.10.2), tem-se:

$$
\operatorname{SQT}_{(a j)}=\operatorname{SQT}\left(x_{1}, x_{2}\right)-U_{T},
$$

onde:

$$
(5.4 .2 . b) U_{T}=\left[\begin{array}{c}
\frac{(b-1)(K-1)}{b K}\left(x_{1}^{2}+x_{2}^{2}-y_{1}^{2}-y_{2}^{2}\right)-2 \frac{\left(K L^{*}+b C_{1}^{*}-G^{*}\right)}{b K}\left(x_{1}-y_{1}\right) \\
-2 \frac{\left(K L+b C_{2}^{*}-G^{*}\right)}{b K}\left(x_{2}-y_{2}\right) \\
-\frac{2(K-1)}{b K}\left(x_{1} x_{2}-y_{1} y_{2}\right)
\end{array}\right]
$$


58.

De 4.10, tem-se:

$$
\operatorname{SQR}_{\left(z_{1}, z_{2}\right)}=\operatorname{SQTotal}_{\left(z_{1}, z_{2}\right)}-\operatorname{SQC}_{\left(z_{1}, z_{2}\right)} .
$$

Tornando minima a $\operatorname{SQR}\left(z_{1}, z_{2}\right)$, obtêm-se as estimativas de um delineamento inteiramente casualizado:

$(5.4 .2 . c)$

$$
\left\{\begin{array}{l}
z_{1}=\frac{C_{1}^{*}}{K-1} \\
z_{2}=\frac{C_{2}^{*}}{k-1}
\end{array}\right.
$$

De (4.10.4) tem-se:

$$
\mathrm{SQL}_{(a j)}=\mathrm{SQL}_{\left(\mathrm{x}_{1}, \mathrm{x}_{2}\right)}-\mathrm{U}_{\mathrm{L}}
$$

onde:

$$
(5.4 .2 . d) U_{L}=\left[\begin{array}{l}
\frac{K-1}{b K}\left(x_{1}^{2}+x_{2}^{2}\right)+\frac{(b-1)(K-1)}{b K}\left(y_{1}^{2}+y_{2}^{2}\right)+\frac{K-1}{K}\left(z_{1}^{2}+z_{2}^{2}\right)+ \\
+2 \frac{\left(K L^{*}-G^{*}\right)}{b K}\left(x_{1}+x_{2}\right)+\frac{2(K-1)}{b K}\left(x_{1} x_{2}-y_{1} y_{2}\right)- \\
-2 \frac{\left(K L^{*}+b_{1}^{*}-G^{*}\right)}{b K} y_{1}-2 \frac{\left(K L^{*}+b_{2}^{*}-G^{*}\right)}{b K} y_{2}
\end{array}\right]
$$


59.

\subsection{3 - Anālise da variāncia}

o quadro da análise da variância è o dado a seguir.

\begin{tabular}{|c|c|c|c|c|c|}
\hline C.V. & G.L. & $S \cdot Q$. & Q.M. & & $\mathrm{F}$ \\
\hline Tratamentos (aj) & $v-1$ & $\mathrm{SQ}(\mathrm{T} \mid \ell, \mathrm{c})=\mathrm{SQT}\left(\mathrm{x}_{1}, \mathrm{x}_{2}\right)^{-\mathrm{U}_{\mathrm{T}}}$ & $\mathrm{QM}(\mathrm{T} \mid \ell, \mathrm{c})$ & (a) & $\mathrm{a} / \mathrm{b}$ \\
\hline Linhas $(a j)$ & $K-1$ & $S Q(L \mid c)=S Q L\left(x_{1}, x_{2}\right)^{-U_{L}}$ & $\mathrm{QM}(\mathrm{L} \mid \mathrm{c})$ & & \\
\hline Colunas & $b-1$ & $\mathrm{SQC}_{\text {(usual) }}$ & QMC (usual) & & \\
\hline Resíduo & $(K-2)-2]$ & ] $\mathrm{SQR}$ & QMR & (b) & \\
\hline Total & $-1)-2]$ & SQTotal (usual) & & & \\
\hline
\end{tabular}

5.4.4 - Variāncia da estimativa de um contraste entre médias de tratamentos, ajustadas para linhas e colu nas

Como dado em (5.3.4), tem-se:

$$
V\left(\hat{E}_{i}-\hat{E}_{i},\right)=P^{\prime} F_{1}^{-1} P \sigma^{2} .
$$


5.5 - Caso de Duas Parcelas Perdidas na Mesma Linha, sendo que Apenas em Uma das Colunas Com Parcela Perdida Figure o Tratamento Correspondente à Parcela Perdida da Outra CoIuna. As Parcelas Perdidas são Ignoradas

Seja um delineamento em Quadrado de Youden, onde 1 , $2,3,4,5,6$ e 7 são os tratamentos.

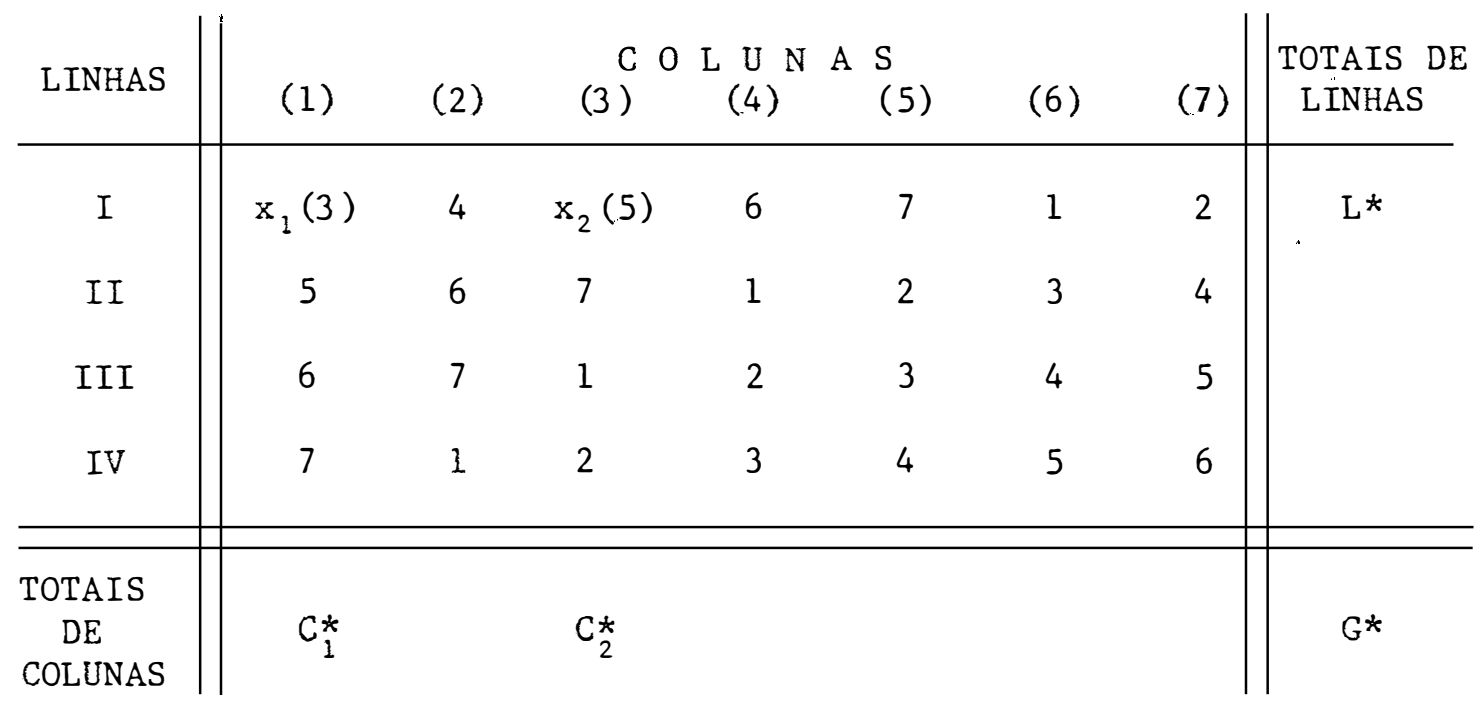

Tem-se: $v=7, \quad K=4, r=4, b=7, \lambda=2$ e $u=I$.

5.5.1 - Sistema de equações normais e estimativas dos parâa metros

0 sistema de equações normais ê o (4.3.a), onde: 
61.

$R_{V V}=\left[\begin{array}{ccccccc}4 & 0 & 0 & 0 & 0 & 0 & 0 \\ 0 & 4 & 0 & 0 & 0 & 0 & 0 \\ 0 & 0 & 3 & 0 & 0 & 0 & 0 \\ 0 & 0 & 0 & 4 & 0 & 0 & 0 \\ 0 & 0 & 0 & 0 & 3 & 0 & 0 \\ 0 & 0 & 0 & 0 & 0 & 4 & 0 \\ 0 & 0 & 0 & 0 & 0 & 0 & 4\end{array}\right] ; E_{b K}=\left[\begin{array}{llll}1 & 1 & 1 & 1 \\ 1 & 1 & 1 & 1 \\ 0 & 1 & 1 & 1 \\ 1 & 1 & 1 & 1 \\ 0 & 1 & 1 & 1 \\ 1 & 1 & 1 & 1 \\ 1 & 1 & 1 & 1\end{array}\right]$

$\mathrm{W}_{\mathrm{vb}}=\left[\begin{array}{ccccccc}0 & 1 & 1 & 1 & 0 & 1 & 0 \\ 0 & 0 & 1 & 1 & 1 & 0 & 1 \\ 0 & 0 & 0 & 1 & 1 & 1 & 0 \\ 0 & 1 & 0 & 0 & 1 & 1 & 1 \\ 1 & 0 & 0 & 0 & 0 & 1 & 1 \\ 1 & 1 & 0 & 1 & 0 & 0 & 1 \\ 1 & 1 & 1 & 0 & 1 & 0 & 0\end{array}\right] ; N_{K K}=\left[\begin{array}{llll}5 & 0 & 0 & 0 \\ 0 & 7 & 0 & 0 \\ 0 & 0 & 7 & 0 \\ 0 & 0 & 0 & 7\end{array}\right]$

$$
\mathrm{B}_{\mathrm{Kb}}=\left[\begin{array}{ccccccc}
0 & 1 & 0 & 1 & 1 & 1 & 1 \\
1 & 1 & 1 & 1 & 1 & 1 & 1 \\
1 & 1 & 1 & 1 & 1 & 1 & 1 \\
1 & 1 & 1 & 1 & 1 & 1 & 1
\end{array}\right]
$$




$$
\mathrm{H}_{\mathrm{bb}}=\left[\begin{array}{ccccccc}
3 & 0 & 0 & 0 & 0 & 0 & 0 \\
0 & 4 & 0 & 0 & 0 & 0 & 0 \\
0 & 0 & 3 & 0 & 0 & 0 & 0 \\
0 & 0 & 0 & 4 & 0 & 0 & 0 \\
0 & 0 & 0 & 0 & 4 & 0 & 0 \\
0 & 0 & 0 & 0 & 0 & 4 & 0 \\
0 & 0 & 0 & 0 & 0 & 0 & 4
\end{array}\right]
$$

Considerando-se as restrições $\sum \hat{c}_{j}=0$ e $\sum \hat{l}_{k}=0$, tem-se as estimativas dos parâmetros, (4.4.b), ou seja:

$(5.5 .1 . \mathrm{a}) \quad \hat{B}=\left[\begin{array}{ccc}{\left[\mathrm{R}_{\mathrm{VV}}\right]} & {\left[\mathrm{E}_{\mathrm{bK}}-\mathrm{A}_{\mathrm{bK}}\right]} & {\left[\mathrm{W}_{\mathrm{vb}}-\mathrm{A}_{\mathrm{Vb}}\right]} \\ {\left[\mathrm{E}_{\mathrm{Kb}}^{\prime}\right]} & {\left[\mathrm{N}_{\mathrm{KK}}\right]} & {\left[\mathrm{B}_{\mathrm{Kb}}-\mathrm{A}_{\mathrm{Kb}}\right]} \\ {\left[\mathrm{W}_{\mathrm{bV}}^{\prime}\right]} & {\left[\mathrm{B}_{\mathrm{bK}}^{\prime}-\mathrm{A}_{\mathrm{bK}}\right]} & {\left[\mathrm{H}_{\mathrm{bb}}\right]}\end{array}\right]\left[\begin{array}{c}\mathrm{T} \\ \mathrm{C}\end{array}\right]$

5.2 .2 - Cálculo das $\operatorname{SQP}_{(1)}, \mathrm{SQP}_{(2)}$ e $\mathrm{SQP}_{(3)}$

A SQP $_{(1)},(4.5 . a)$, é obtida com a solução (5.5.1.a). Para determinar-se a SQP (2) utiliza-se a solução (4.5.g), que para o caso é dada por: 


$$
\tilde{B}^{*}=\left[\begin{array}{ccccccccccc}
5 & 0 & 0 & 0 & -1 & 0 & -1 & 0 & 0 & 0 & 0 \\
0 & 7 & 0 & 0 & 0 & 0 & 0 & 0 & 0 & 0 & 0 \\
0 & 0 & 7 & 0 & 0 & 0 & 0 & 0 & 0 & 0 & 0 \\
0 & 0 & 0 & 7 & 0 & 0 & 0 & 0 & 0 & 0 & 0 \\
0 & 1 & 1 & 1 & 3 & 0 & 0 & 0 & 0 & 0 & 0 \\
1 & 1 & 1 & 1 & 0 & 4 & 0 & 0 & 0 & 0 & 0 \\
0 & 1 & 1 & 1 & 0 & 0 & 3 & 0 & 0 & 0 & 0 \\
1 & 1 & 1 & 1 & 0 & 0 & 0 & 4 & 0 & 0 & 0 \\
1 & 1 & 1 & 1 & 0 & 0 & 0 & 0 & 4 & 0 & 0 \\
1 & 1 & 1 & 1 & 0 & 0 & 0 & 0 & 0 & 4 & 0 \\
1 & 1 & 1 & 1 & 0 & 0 & 0 & 0 & 0 & 0 & 4
\end{array} \mathrm{~L}_{3} \mathrm{~L}_{4}\right.
$$

A SQP $_{(3)},(4.5 . n)$, é determinada com a solução:

$$
\underline{\underline{c}}=\mathrm{H}^{-1} \mathrm{C} \text {. }
$$

\subsection{3 - Anālise da variāncia}

\begin{tabular}{|c|c|c|c|c|}
\hline C.V. & G.L. & $S \cdot Q$. & Q.M. & $\mathrm{F}$ \\
\hline Tratamentos $(a j)$ & $v-1=6$ & $\mathrm{SQ}(\mathrm{T} \mid 2, \mathrm{C})=\mathrm{SQP}(1)^{-\mathrm{SQP}}(2)$ & $\mathrm{QM}(\mathrm{T} \mid \ell, \mathrm{c}) \quad(\mathrm{a})$ & $a / b$ \\
\hline Linhas (aj) & $K-1=3$ & $S Q(L \mid c)=S Q P(2)^{-S Q P}(3)$ & $\mathrm{QM}(\mathrm{L} \mid \mathrm{c})$ & \\
\hline Colunas & $b-1=6$ & $\mathrm{SQC}$ (usual) & QMC (usual) & \\
\hline Residuo & $(K-2)-2]=10$ & $S Q R$ & QMR $\quad$ (b) & \\
\hline Total & $(k-1)-2]=25$ & SQTotal (usual) & & \\
\hline
\end{tabular}

o quadro da anālise da variância é o dado a seguir. 
A $S Q(T \mid \ell, c)$ e a $S Q(L \mid c)$ podem ser obtidas tambëm por $(4.11 .1 . b)$ e $(4.11 .2 . e)$, respectivamente.

5.5.4 - Variāncia da estimativa de um contraste entre mēdias de tratamentos, ajustadas para linhas e colunas

Como visto em (4.13.e), tem-se:

$$
V\left(\hat{E}_{i}-\hat{E}_{i}^{\prime}\right)=P^{\prime} F_{1}^{-1} P \sigma^{2},
$$

onde $\mathrm{F}_{1}^{-1}$ é dado em $(5.5 \cdot 1 . \mathrm{a})$, e QMR $\doteq \sigma^{2}$.

5.6 - Caso de Duas Parcelas Perdidas na Mesma Linha, sendo que Ape nas em Uma das Colunas com Parcela Perdida Figure o Tratamen to Correspondente à Parcela Perdida da Outra Coluna. As Estī mativas das Parcelas Perdidas são Consideradas

5.6.1 - Fórmulas para as estimativas das parcelas perdidas

Minimizando-se a $\mathrm{SQR}_{\left(\mathrm{x}_{1}, \mathrm{x}_{2}\right)}$, dada em 4.9, obtēm-se um sistema de equações determinado, cuja solução são as estimativas das parcelas perdidas:

$$
\begin{aligned}
& K(K-1)(K-2) x_{1}-[(\lambda-1)(K-1)+u] x_{2}=\left[\begin{array}{l}
\lambda K L^{*}+\lambda b C_{1}^{*}+K(K-1) T_{1}^{*}- \\
-\lambda G^{*}-K T_{1}^{\prime}-(K-1) Q_{1}+Q_{1}^{\prime}
\end{array}\right] \\
& K(K-1)(K-2) x_{2}-[(\lambda-1)(K-1)+u] x_{1}=\left[\begin{array}{c}
\lambda K L^{*}+\lambda b C_{2}^{*}+K(K-1) T_{2}^{*}- \\
-\lambda G^{*}-K T_{2}^{\prime}-(K-1) Q_{2}+Q_{2}^{\prime}
\end{array}\right]
\end{aligned}
$$


5.6.2 - Förmulas para as correções das somas de quadrados de tratamentos e de linhas: $U_{T}$ e $U_{L}$

Minimizando-se a $\mathrm{SQR}\left(\mathrm{y}_{\frac{1}{2}}, \mathrm{y}_{2}\right)$, dada em 4.10, obtém-se um sistema de equações, cuja solução são as estimativas das parcelas perdidas de um delineamento em blocos casualizados:

$(5.6 .2 . a)$

$$
\begin{aligned}
& (b-1) y_{1}-y_{2}=\frac{K L^{*}+b C_{1}^{*}-G^{*}}{K-1} \\
& (b-1) y_{2}-y_{1}=\frac{K L^{*}+b C_{2}^{*}-G^{*}}{K-1}
\end{aligned}
$$

Como dado em $(4.10 .2)$, tem-se:

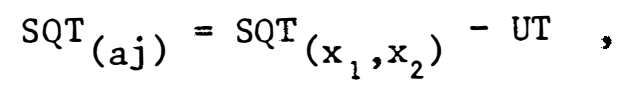

onde:

$(5.6 .2 . b)$

$$
U_{T}=\left[\begin{array}{c}
\frac{(b-1)(K-1)}{b K}\left(x_{1}^{2}+x_{2}^{2}-y_{1}^{2}-y_{2}^{2}\right)- \\
-2 \frac{K L^{*}+b C_{1}^{*}-G^{*}}{b K}\left(x_{1}-y_{1}\right)- \\
-2 \frac{K L^{*}+b C_{2}^{*}-G^{*}}{b K}\left(x_{2}-y_{2}\right)- \\
-2 \frac{(K-1)}{b K}\left(x_{1} x_{2}-y_{1} y_{2}\right)
\end{array}\right]
$$


Tornando mínima a $\mathrm{SQR}_{\left(z_{1}, z_{2}\right)}$, dada em 4.10, obtêm-se as estimativas das parcelas perdidas de um delineamento inteiramente casualizado:

$(5.6 .2 . c)$

$$
0 \mid \begin{aligned}
& z_{1}=\frac{C_{1}^{*}}{K-1} \\
& z_{2}=\frac{C_{2}^{*}}{K-1}
\end{aligned}
$$

De $(4.10 .4)$, tem-se:

$$
S_{(a j)}=S Q L\left(x_{1}, x_{2}\right)-U_{L}
$$

onde:

$$
(5.6 .2 . d) \quad U_{L}=\left[\begin{array}{l}
\frac{(K-1)}{b K}\left(x_{1}^{2}+x_{2}^{2}\right)+\frac{(b-1)(K-1)}{b K}\left(y_{1}^{2}+y_{2}^{2}\right)+ \\
+\frac{K-1}{K}\left(z_{1}^{2}+z_{2}^{2}\right)+2 \frac{\left(K L *-G^{*}\right)}{b K}\left(x_{1}+x_{2}\right)+ \\
+\frac{2(K-1)}{b K}\left(x_{1} x_{2}-y_{1} y_{2}\right)-2 \frac{\left(K L *+b C_{1}^{*}-G^{*}\right)}{b K} y_{1} \\
-2 \frac{\left(K L *+b C_{2}^{*}-G^{*}\right)}{b K} y_{2}
\end{array}\right]
$$


5.6.3 - Anālise da variância

0 quadro da anâlise da variância ê o dado a seguir.

\begin{tabular}{|c|c|c|c|c|}
\hline C.v. & G.L. & S.Q. & Q.M. & F \\
\hline Tratamentos $(a j)$ & $v-1$ & $Q(T \mid \ell, c)=S Q T\left(x_{1}, x_{2}\right)^{-U} T$ & $\mathrm{QM}(\mathrm{T} \mid \ell, \mathrm{c}) \quad(\mathrm{a})$ & $a / b$ \\
\hline Linhas $(a j)$ & $\mathrm{K}-1$ & $S Q(L \mid c)=S Q L\left(x_{1}, x_{2}\right)^{-U L}$ & $Q M(L \mid c)$ & \\
\hline Colunas & $b-1$ & $\mathrm{SQC}_{\text {(usual) }}$ & QMC (usual) & \\
\hline Resỉduo & $(K-2)-2]$ & $\mathrm{SQR}$ & QMR & \\
\hline Total & $K-1)-2]$ & SQTotal (usual) & & \\
\hline
\end{tabular}

5.6.4 - Variância da estimativa de um contraste entre médias de tratamentos ajustadas para linhas e colunas

Como dado em (5.5.4), tem-se:

$$
V\left(\hat{E}_{i}-\hat{E}_{i}^{\prime}\right)=P^{\prime} F_{1}^{-1} P \sigma^{2}
$$

5.7 - Caso de Duas Parcelas Perdidas na Mesma Linha, sendo que em Cada Uma das Colunas com Parcela Perdida Figure o Tratamento Correspondente à Parcela Perdida da Outra Coluna e Vice-Versa. As Parcelas São Ignoradas.

Seja um delineamento em quadrado de Youden, onde A, B, C, D e E são os tratamentos: 


\begin{tabular}{c||ccccc||c}
$\therefore$ LINHAS & \multicolumn{7}{|c}{ C O L U N A S } & TOTAIS \\
\hline I & $(1)$ & $(2)$ & $(3)$ & $(4)$ & (5) & DE LINHAS \\
II & $\mathrm{x}_{1}(\mathrm{D})$ & $\mathrm{A}$ & $\mathrm{B}$ & $\mathrm{x}_{2}(\mathrm{C})$ & $\mathrm{E}$ & $\mathrm{L}^{*}$ \\
$\mathrm{C}$ & $\mathrm{E}$ & $\mathrm{A}$ & $\mathrm{B}$ & $\mathrm{D}$ & \\
$\mathrm{III}$ & $\mathrm{E}$ & $\mathrm{B}$ & $\mathrm{C}$ & $\mathrm{D}$ & $\mathrm{A}$ & \\
\hline $\begin{array}{c}\text { TOTAIS DE } \\
\text { COLUNAS }\end{array}$ & $\mathrm{B}$ & $\mathrm{D}$ & $\mathrm{E}$ & $\mathrm{A}$ & $\mathrm{C}$ & \\
\hline
\end{tabular}

Tem-se: $v=5, k=4, r=4, b=5, \lambda=3$ e $u=1$.

5.7.1 - Sistema de equações normais e estimativas dos parâmetros

O sistema de equações normais é o (4.3.a), onde:

$$
\begin{aligned}
& R_{V V}=\left[\begin{array}{lllll}
4 & 0 & 0 & 0 & 0 \\
0 & 4 & 0 & 0 & 0 \\
0 & 0 & 3 & 0 & 0 \\
0 & 0 & 0 & 3 & 0 \\
0 & 0 & 0 & 0 & 4
\end{array}\right] ; E_{b K}=\left[\begin{array}{llll}
1 & 1 & 1 & 1 \\
1 & 1 & 1 & 1 \\
0 & 1 & 1 & 1 \\
0 & 1 & 1 & 1 \\
1 & 1 & 1 & 1
\end{array}\right] ; \\
& N_{K K}=\left[\begin{array}{llll}
3 & 0 & 0 & 0 \\
0 & 5 & 0 & 0 \\
0 & 0 & 5 & 0 \\
0 & 0 & 0 & 5
\end{array}\right] ; \mathrm{W}_{\mathrm{Vb}}=\left[\begin{array}{ccccc}
0 & 1 & 1 & 1 & 1 \\
1 & 1 & 1 & 1 & 0 \\
1 & 0 & 1 & 0 & 1 \\
0 & 1 & 0 & 1 & 1 \\
1 & 1 & 1 & 0 & 1
\end{array}\right] ;
\end{aligned}
$$


69.

$$
\mathrm{B}_{\mathrm{Kb}}=\left[\begin{array}{ccccc}
0 & 1 & 1 & 0 & 1 \\
1 & 1 & 1 & 1 & 1 \\
1 & 1 & 1 & 1 & 1 \\
1 & 1 & 1 & 1 & 1
\end{array}\right] ; \mathrm{Hbb}_{\mathrm{b}}=\left[\begin{array}{ccccc}
3 & 0 & 0 & 0 & 0 \\
0 & 4 & 0 & 0 & 0 \\
0 & 0 & 4 & 0 & 0 \\
0 & 0 & 0 & 3 & 0 \\
0 & 0 & 0 & 0 & 4
\end{array}\right] ;
$$

$$
\text { Considerando-se as restrições } \sum \hat{\mathrm{c}}_{\mathrm{j}}=0 \text { e } \Sigma \mathbb{l}_{k}=0 \text {, têm- }
$$

-se as estimativas dos parâmetros, (4.4.b), ou seja:

$$
(5.7 .1 . \mathrm{a}) \quad \widehat{B}=\left[\begin{array}{ccc}
{\left[\mathrm{R}_{\mathrm{VV}}\right]} & {\left[\mathrm{E}_{\mathrm{bK}}-\mathrm{A}_{\mathrm{bK}}\right]} & {\left[\mathrm{W}_{\mathrm{vb}}-\mathrm{A}_{\mathrm{vb}}\right]} \\
{\left[\mathrm{E}_{\mathrm{Kb}}^{\prime}\right]} & {\left[\mathrm{N}_{\mathrm{KK}}\right]} & {\left[\mathrm{B}_{\mathrm{Kb}}-\mathrm{A}_{\mathrm{Kb}}\right]} \\
{\left[\mathrm{W}_{\mathrm{bV}}^{\prime}\right]} & {\left[\mathrm{B}_{\mathrm{bK}}^{\prime}-\mathrm{A}_{\mathrm{bK}}\right]} & {\left[\mathrm{H}_{\mathrm{bb}}\right]}
\end{array}\right]^{-1}\left[\begin{array}{c}
\mathrm{T} \\
\mathrm{C}
\end{array}\right]
$$

5.7 .2 - Cálculo das $\operatorname{SQP}_{(1)}, \mathrm{SQP}_{(2)}$ e $\operatorname{SQP}_{(3)}$

$$
\operatorname{A~SQP}_{(1)},(4.5 . a), \bar{e} \text { obtida com a solução (5.7.1.a). }
$$

Para determinar-se a SQP (2) utiliza-se a solução (4.5.g), que para o caso é dada por: 
$\tilde{\beta}^{*}=\left[\begin{array}{ccccccccc}3 & 0 & 0 & 0 & -1 & 0 & 0 & -1 & 0 \\ 0 & 5 & 0 & 0 & 0 & 0 & 0 & 0 & 0 \\ 0 & 0 & 5 & 0 & 0 & 0 & 0 & 0 & 0 \\ 0 & 0 & 0 & 5 & 0 & 0 & 0 & 0 & 0 \\ 0 & 1 & 1 & 1 & 3 & 0 & 0 & 0 & 0 \\ 1 & 1 & 1 & 1 & 0 & 4 & 0 & 0 & 0 \\ 1 & 1 & 1 & 1 & 0 & 0 & 4 & 0 & 0 \\ 0 & 1 & 1 & 1 & 0 & 0 & 0 & 3 & 0 \\ 1 & 1 & 1 & 1 & 0 & 0 & 0 & 0 & 4\end{array}\right]^{-1} \quad\left[\begin{array}{c}L_{*} \\ L_{2} \\ L_{3} \\ L_{4} \\ C_{1}^{*} \\ C_{3} \\ C_{4} \\ C_{2}^{*} \\ C_{5}\end{array}\right]$

$\operatorname{A~SQP~}_{(3)},(4.5 . n)$, é determinada com a solução:

$$
\underline{\underline{c}}=H^{-1} \mathrm{C} \text {. }
$$

5.7.3 - Anālise da variāncia

o quadro da anālise da variância é o dado a seguir.

\begin{tabular}{|c|c|c|c|c|}
\hline C.V. & G.L. & S.Q. & Q.M. & $\mathrm{F}$ \\
\hline Tratamentos $(a j)$ & $v-1=4$ & $\mathrm{SQ}(\mathrm{T} \mid \ell, \mathrm{C})=\mathrm{SQP}(1)^{-\mathrm{SQP}}(2)$ & $\mathrm{QM}(\mathrm{T} \mid \ell, \mathrm{c}) \quad(\mathrm{a})$ & $a / b$ \\
\hline Linhas $(a j)$ & $k-1=3$ & $\mathrm{SQ}(L \mid c)=S Q P(2)^{-S Q P}(3)$ & $\mathrm{QM}(\mathrm{L} \mid \mathrm{C})$ & \\
\hline Colunas & $b-1=4$ & $\mathrm{SQC}_{\text {(usual) }}$ & QMC (usual) & \\
\hline Resíduo & $(K-2)-2]=6$ & $5 \mathrm{SQR}$ & QMR & \\
\hline Total & $-1)-2]=17$ & SQTotal (usual) & & \\
\hline
\end{tabular}


A $S Q(T \mid 2, C)$ e a $S Q(L \mid C)$ podem ser obtidas também por $(4.11 .1 . b)$ e $(4.11 .2 . e)$, respectivamente.

5.7.4 - Variāncia da estimativa de um contraste entre médias de tratamentos, ajustadas para linhas e colunas

$$
\begin{aligned}
& \text { Como visto em }(4.13 . e) \text {, tem-se: } \\
& \qquad V\left(\hat{t}_{i}-\hat{t}_{i^{\prime}}\right)=P^{\prime} F^{-1} P \sigma^{2},
\end{aligned}
$$

onde $F_{1}^{-1}$ é dado em $(5 \cdot 7.1 . a)$ e QMR $\doteq \sigma^{2}$.

5.8 - Caso de Duas Parcelas Perdidas na Mesma Linha, sendo que em Cada Uma das Colunas com Parcela Perdida Figura o Tratamento Correspondente à Parcela Perdida da Outra Coluna e Vice- Versa. As Estimativas das Parcelas Perdidas são Consideradas

5.8.1 - Förmulas para as estimativas das parcelas perdidas

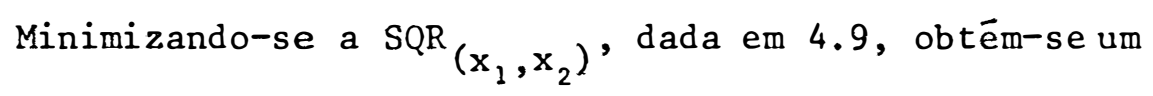
sistema de equações determinado, cuja solução são as estimativas das parcelas perdidas: 
$K(K-1)(K-2) x_{1}-[(\lambda-2)(K-1)+u] \quad x_{2}=\left[\begin{array}{l}\lambda K L^{*}+\lambda b C_{1}^{*}+K(K-1) T_{1}^{*} \\ -\lambda G *-K T_{1}^{\prime}-(K-1) Q_{1}+Q_{1}^{*}\end{array}\right]$
$K(K-1)(K-2) x_{2}-[(\lambda-2)(K-1)+u] \quad x_{1}=\left[\begin{array}{ll}\lambda K L *+\lambda b C_{2}^{*}+K(K-1) T_{2}^{*}- \\ -\lambda G^{*}-K T_{1}^{\prime}-(K-1) Q_{2}+Q_{2}^{\prime}\end{array}\right]$

$$
(5.8 .1 . a)
$$

5.8.2 - Förmulas para as correções das somas de quadrados de tratamentos e de linhas: $U_{T}$ e $U_{L}$

Minimizando-se a $\operatorname{SQR}_{\left(\mathrm{y}_{1}, \mathrm{y}_{2}\right)}$, dada em 4.10, obtém-se um sistema de equações, cuja solução são as estimativas das parcelas perdidas de um delineamento em blocos casualizados:

$(5.8 .2 . a)$

$$
\begin{aligned}
& \mid \begin{array}{l}
(b-1) y_{1}-y_{2}=\frac{K L^{*}+b C_{1}^{*}-G^{*}}{K-1} \\
(b-1) y_{2}-y_{1}=\frac{K L^{*}+b C_{2}^{*}-G^{*}}{K-1}
\end{array} \\
& \text { Como dado em }(4 \cdot 10.2) \text {, tem-se: } \\
& \mathrm{SQT}_{(a j)}=\mathrm{SQT}_{\left(\mathrm{x}_{1}, \mathrm{x}_{2}\right)}-\mathrm{U}_{\mathrm{T}} \text {, }
\end{aligned}
$$

onde: 
$(5.8 .2 . b)$

$$
U_{T}=\left[\begin{array}{l}
\frac{(b-1)(K-1)}{b K}\left(x_{1}^{2}+x_{2}^{2}-y_{1}^{2}-y_{2}^{2}\right)- \\
-2 \frac{K L^{*}+b C_{1}^{*}-G^{*}}{b K}\left(x_{1}-y_{1}\right)- \\
-2 \frac{K L^{*}+b C_{2}^{*}-G^{*}}{b K}\left(x_{2}-y_{2}\right)- \\
-2 \frac{(K-1)}{b K}\left(x_{1} x_{2}-y_{1} y_{2}\right)
\end{array}\right]
$$

Minimizando-se a $\mathrm{SQR}_{\left(z_{1}, z_{2}\right)}$, dada em 4.10, obtêm-se as estimativas das parcelas perdidas de um delineamento inteiramente casualizado:

$(5.8 .2 . c)$

$$
\left\{\begin{array}{l}
z=\frac{C_{1}^{*}}{K-1} \\
z=\frac{C_{2}^{*}}{k-1}
\end{array}\right.
$$

De $(4.10 .4)$, tem-se:

$$
\operatorname{SQL}_{(a j)}=\operatorname{SQL}_{\left(x_{1}, x_{2}\right)}-U_{L},
$$

onde: 
$(5.8 .2 . d) \quad U_{L}=\left[\begin{array}{l}\frac{(K-1)}{b K}\left(x_{1}^{2}+x_{2}^{2}\right)+\frac{(b-1)(K-1)}{b K}\left(y_{1}^{2}+y_{2}^{2}\right)+ \\ +\frac{K-1}{K}\left(z_{1}^{2}+z_{2}^{2}\right)+2 \frac{\left(K L *-G^{*}\right)}{b K}\left(x_{1}+x_{2}\right)+ \\ +2 \frac{(K-1)}{b K}\left(x_{1} x_{2}-y_{1} y_{2}\right)-2 \frac{\left(K L *+b C_{1}^{*}-G^{*}\right)}{b K} y_{1}\end{array}\right]$

5.8.3 - Anālise da variāncia

o quadro da análise da variância é o dado a seguir:

\begin{tabular}{|c|c|c|c|c|}
\hline C.V. & G.L. & $S \cdot Q$. & Q.M. & $\mathrm{F}$ \\
\hline Tratamentos $(a j)$ & $v-1 \quad s$ & 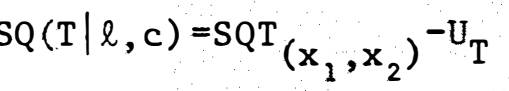 & $\mathrm{QM}(\mathrm{T} \mid \ell, \mathrm{c}) \quad(\mathrm{a})$ & $a / b$ \\
\hline $\operatorname{Linhas}(a j)$ & $\mathrm{K}-1$ & $S Q(L \mid c)=S Q L\left(x_{1}, x_{2}\right)^{-U_{L}}$ & $\mathrm{QM}(\mathrm{L} \mid \mathrm{c})$ & \\
\hline Colunas & $b-1$ & $\mathrm{SQC}_{\text {(usual) }}$ & $\mathrm{QMC}_{\text {(usual) }}$ & \\
\hline Resíduo & 1) $(\mathrm{K}-2)-2]$ & $\mathrm{SQR}$ & QMR & \\
\hline Total & $(\mathrm{bK}-1)-2]$ & SQTotal (usual) & & \\
\hline
\end{tabular}

5.8.4 - Variāncia da estimativa de um contraste entre médias de tratamentos ajustadas para linhas e colunas

Como dado em (5.7.4), tem-se:

$$
V\left(\hat{E}_{i}-\hat{t}_{i}{ }^{\prime}\right)=P^{\prime} F_{1}^{-1} P \sigma^{2} \text {. }
$$


5.9 - Exemplos Numéricos

Para ilustrar os resultados obtidos neste trabalho, serão usados os experimentos apresentados em 3, onde as corretas somas de quadrados serão obtidas:

a) diretamente através de matrizes $\left[\mathrm{SQ}_{(\mathrm{a})}\right]$;

b) através das fórmulas deduzidas $\left[\mathrm{SQ}_{(b)}\right]$.

5.9.1 - Caso de uma parcela perdida $\left(y_{114}\right)$

Considerando-se os dados da Tabela 1, tem-se:

$$
\begin{aligned}
x & =512,1666667 \\
y & =590,75 \\
z & =569,00 \\
\mathrm{SQP}_{(1)} & =4.433 .810,437 \\
\mathrm{SQP}_{(2)} & =4.342 .808,182 \\
\mathrm{SQP}_{(3)} & =4.311 .473,250 \\
\mathrm{U}_{\mathrm{T}} & =3.705,204164 \\
\mathrm{U}_{\mathrm{L}} & =-1.282,683405
\end{aligned}
$$


Anālise da variāncia

\begin{tabular}{lcrccc}
\hline C.V. & G.L. & S.Q. $(a)$ & S.Q. $(b)$ & Q.M. & F \\
\hline Tratam. $(a j)$ & 4 & $91.002,245$ & $91.002,245$ & $22.750,5613$ & $6,93 *$ \\
Linhas $(a j)$ & 3 & $31.334,932$ & $31.334,932$ & $10.444,9773$ & \\
Colunas & 4 & $42.629,25$ & $42.629,25$ & $10.657,3125$ & \\
Resíduo & 7 & $22.987,573$ & $22.987,573$ & $3.283,9290$ & \\
\hline Total & 18 & $187.954,00$ & $187.954,00$ & & \\
\hline
\end{tabular}

5.9.2 - Caso de duas parcelas perdidas na mesma linha sendo que 0 tratamento correspondente $\bar{a}$ parcela perdida de uma coluna não figure dentre as parcelas remanes centes da outra coluna em que ocorreu a perda e vice-versa $\left(y_{117}\right.$ e $\left.y_{132}\right)$

Considerando-se os dados da Tabela 2, tem-se:

$$
\begin{array}{rrr}
x_{1}=23,60 & ; & x_{2}=35,30 \\
y_{1}=24,30 \quad & y_{2}=34,75 \\
z_{1}=18,80 \quad & z_{2}=29,25 \\
\operatorname{SQP}_{(1)} & =18.353,25142 \\
\operatorname{SQP}_{(2)} & =18.303,54809 \\
\operatorname{SQP}_{(3)} & =18.200,22833 \\
U_{T} & =0,526191 \\
U_{L} & =39,235476
\end{array}
$$


Ânālise da variāncia

\begin{tabular}{lrrrrr}
\hline C.V. & G.L. & S.Q.(a) & S.Q. $(b)$ & Q.M. & F \\
\hline Tratam. $(a j)$ & 6 & 49,70333 & 49,70333 & 8,2839 & 0,39 \\
Linhas $(a j)$ & 2 & 103,31976 & 103,31976 & 51,6599 & \\
Colunas & 6 & 738,33359 & 738,33359 & 123,0556 & \\
Resíduo & 4 & 84,74858 & 84,74858 & 21,1871 & \\
\hline Total & 18 & 976,10526 & 976,10526 & & \\
\hline
\end{tabular}

5.9.3 - Caso de duas parcelas perdidas na mesma linha, sendo que apenas em uma das colunas com parcela perdida figure o tratamento correspondente à parcela da outra coluna $\left(y_{114}\right.$ e $\left.y_{121}\right)$

Considerando-se os dados da Tabela 1, tem-se:

$$
\begin{array}{rlc}
\mathrm{x}_{1}=530,625 & ; & \mathrm{x}_{2}=573,375 \\
\mathrm{y}_{1}=576,666667 & ; & \mathrm{y}_{2}=461,66667 \\
\mathrm{z}_{1}=569,00 & ; & \mathrm{z}_{2}=454,00 \\
\mathrm{SQP}(1) & =4.166 .576,508 \\
\mathrm{SQP} & (2)=4.076 .268,649 \\
\mathrm{SQP}_{(3)} & =4.046 .221,250 \\
\mathrm{U}_{\mathrm{T}} & =10.302,123420 \\
\mathrm{U}_{\mathrm{L}} & =1.490,1499
\end{array}
$$


Anālise da variāncia

\begin{tabular}{lccccc}
\hline C.V. & G.L. & S.Q. $(\mathrm{a})$ & S.Q. (b) & Q.M. & F \\
\hline Tratam. $(\mathrm{aj})$ & 4 & $90.307,8590$ & $90.307,8590$ & $22.576,9648$ & $6,19 *$ \\
Linhas $(\mathrm{aj})$ & 3 & $30.047,3990$ & $30.047,3990$ & $10.015,7997$ & \\
Colunas & 4 & $43.657,6940$ & $43.657,6940$ & $10.914,4235$ & \\
Resíduo & 6 & $21.897,4920$ & $21.897,4920$ & $3.659,5820$ & \\
\hline Total & 17 & $185.910,4440$ & $185.910,4440$ & & \\
\hline
\end{tabular}

5.9.4 - Caso de duas parcelas perdidas na mesma linha, sendo que em cada uma das colunas com parcela perdida figure o tratamento correspondente à parcela perdida da outra coluna e vice-versa $\left(y_{114}\right.$ e $\left.y_{143}\right)$

Considerando-se os dados da Tabela 1, tem-se:

$$
\begin{array}{lll}
\mathrm{x}_{1}=513,20 & ; & \mathrm{x}_{2}=589,20 \\
\mathrm{y}_{1}=551,777778 & ; & \mathrm{y}_{2}=427,111111 \\
\mathrm{z}_{1}=569 & ; & \mathrm{z}_{2}=444,333333
\end{array}
$$

$$
\begin{aligned}
\mathrm{SQP}_{(1)} & =4.093 .935,676 \\
\mathrm{SQP}_{(2)} & =4.016 .588,126 \\
\mathrm{SQP}_{(3)} & =3.986 .005,583 \\
\mathrm{U}_{\mathrm{T}} & =18.532,539230 \\
\mathrm{U}_{\mathrm{L}} & =-457,549340
\end{aligned}
$$


Anālise da variāncia

\begin{tabular}{lccccc}
\hline C.V. & G.L. & S.Q. $(a)$ & S.Q. $_{(b)}$ & Q.M. & F \\
\hline Tratam. $(a j)$ & 4 & $77.347,5500$ & $77.347,5500$ & $19.336,8875$ & $5,05 *$ \\
Linhas $(a j)$ & 3 & $30.582,5430$ & $30.582,5430$ & $10.194,1810$ & \\
Colunas & 4 & $44.509,5270$ & $44.509,5270$ & $11.127,3818$ & \\
Residuo & 6 & $22.973,3240$ & $22.973,3240$ & & \\
\hline Total & 17 & $175.412,9440$ & $175.412,9440$ & & \\
\hline
\end{tabular}

Embora a demonstração não esteja presente, estudaram-se os componentes de variância e constatou-se, como é usual ocorrer, que a SQR perde tantos graus de liberdade quantas forem as parcelas perdidas. 
80.

\section{CONCLUSÖES}

De um modo geral, conclui-se:

6.1 - As corretas somas de quadrados podem ser obtidas:

a) diretamente atravēs de matrizes;

b) através de fórmulas deduzidas.

6.2 - Para o caso de uma parcela perdida, a correção para SQT (x), $\mathrm{U}_{\mathrm{T}}$, e para a $\mathrm{SQL}_{(\mathrm{x})}, \mathrm{U}_{\mathrm{L}}$, são:

$$
\begin{gathered}
U_{T}=\frac{(b-1)(K-1)}{b k}(x-y)^{2} ; \\
U_{L}=\frac{K-1}{b K}|| x^{2}-(b-1) y+b z^{2}\left|+2 x \frac{\left(K L^{*}-G^{*}\right)}{K-1}\right|
\end{gathered}
$$


Analisando-se a expressão $U_{T}$, verifica-se que é sempre positiva, o que indica que a $\mathrm{SQT}_{(\mathrm{x})}$ está sempre superestimada.

6.3 - A expressão para a correção da $\mathrm{SQT}_{\left(\mathrm{x}_{1}, \mathrm{x}_{2}\right)}, \mathrm{U}_{\mathrm{T}}$, é a mesma para todos os casos estudados de duas parcelas perdidas na mesma linha. O mesmo ocorre para a correção da $S_{\left(x_{1}, x_{2}\right)}, U_{L} \cdot 0$ que diferencia os casos são as fórmulas para as estimativas das parcelas perdidas.

6.4 - Como a SQT é ajustada para linhas e colunas, e a SQL é ajustada para colunas, então a SQC é a usual.

6.5 - A SQR pode ser obtida por diferença entre a SQTotal (usual) e as demais corretas somas de quadrados.

6.6 - Só se chegou a uma única maneira de se obter a variância entre médias de tratamentos ajustadas para linhas e colunas, ou seja, através de matrizes. 
82.

\section{BIBLIOGRAFIA}

ALLAN, F.E. d J. WISHART, 1930. A method of estimating the yield of a missing plot in field experimental work. Jour. Agr. Sci., Cambridge, 20: 399-406.

ANDERSON, R.L., 1946. Missing-plot techniques. Biometrics, Raleigh, 2: $41-57$.

CAMPOS, H., 1964. Estudo sobre análise de experimentos com parcelas perdidas. Piracicaba, ESALQ/USP. 84 p. (Tese de Doutoramento).

CHAKRABARTI, M.C., 1962. Mathematics of Design and Analysis of Experiments. Asia Publishing House, Londres, 120 p.

CLARKE, G.M., 1963. A second set of treatments in a Youden Square design. Biometrics, Raleigh, 19: 98-104.

CLARKE, G.M., 1967. Four-way Balanced Designs based on Youden Squares with 5, 6 or 7 treatments. Biometrics, Raleigh, 23: 803-812. 
COCHRAN, W.G. e G.M. Cox, 1957. Experimental Design. 2. ed., John Wiley, Nova York. 611 p.

FABRE SANCHES, S., 1978. Análise de um experimento em quadrado latí no com perda de parcelas, linha, coluna ou tratamento. Piracicaba, ESALQ/USP. 100 p. (Dissertação de Mestrado).

FABRE SANCHES, S., 1980. Anālise de experimentos em parcelas subdivididas em blocos casualizados, com perda de uma ou duas subparce las ou parcelas. Piracicaba, ESALQ/USP. 119 p. (Tese de Doutoramento).

FINNEY, D.J., 1946. A note on missing-plot techniques. Biometrics, Raleigh, 2: 94 .

HADDAD, M.L., 1978. Estudo sobre parcela perdida em delineamento em parcelas subdivididas. Piracicaba, ESALQ/USP. 53 p. (Dissertação de Mestrado).

KEMPTHORNE, O., 1952. The Design of Experiments. John Willey, Nova York.

PANSE, V.G. e P.V. SUKTHAME, 1957. Statistical methods of agricultural works. New Delhi, Indian Council of Agricultural Research, $361 \mathrm{p}$.

PIMENTEL GOMES, F., 1978. Curso de Estatística Experimental. Livraria Nobel, Piracicaba. 430 p.

PINHO, S.Z., 1973. Observações perdidas em delineamentos em parcelas subdivididas e sub-subdivididas. Botucatu, UNESP. $173 \mathrm{p}$. (Tese de Doutoramento). 
PINHO, S.Z., 1975. Estudo sobre os componentes de variância de um delineamento em parcelas subdivididas com uma parcela perdida. Piracicaba, ESALQ/USP. (Dissertação de Mestrado).

RIBEIRO, V.Q., 1980. Análise de ensaios em parcelas subdivididas com observações perdidas. Piracicaba, ESALQ/USP. 85 p. (Dissertação de Mestrado).

SMITH, C.A.B. e H.O. HARTHEY, 1948. The construction of Youden Squares. Journ. Roy. Stat., Londres, 13: 10.

YOUDEN, W.J., 1937. Use of incomplete block repheations in estimating tabaco-mosaic. Contr. Boyce Thompson Inst., Menasha, 9: 41-48.

YOUDEN, W.J., 1940. Experimental design to increase accuracy of green-house studies. Contr. Boyce Thompson Inst., Menasha, 11: 219-228.

YATES, F., 1933. The analysis of replicated experiments when the fields results are incomplets. Empire Journal Exp. Agr., Oxford, 1: 129-142.

YATES, F., 1936. Incomplete latin square. Journ. Agr. Sci., Cambridge, 26: $301-315$. 